Chapter 8

\title{
Identification of Communication Channels for Remote Sensing Systems Using Volterra Model in Frequency Domain
}

\author{
Vitaliy Pavlenko and Viktor Speranskyy \\ Additional information is available at the end of the chapter \\ http://dx.doi.org/10.5772/58354
}

\section{Introduction}

The sensors in remote sensing systems are looking through a layer of atmosphere separating the sensors from the Earth's surface being observed. It is essential to understand the effects of atmosphere on the electromagnetic radiation travelling from the Earth to the sensor through the atmosphere. The atmospheric constituents cause wavelength dependent absorption and scattering of radiation due to environment interactions, emissions and so on (fig. 1) [6]. The atmosphere between radiating surface and sensor can be understood as communication channel (CC). The technical conditions of CC during operation should be considered for the effective communications. Changes during data transfer can decrease the rate of data transmission in digital CC up to stop of transmission. In analog CC it can be cause distortions and noise of the transmitted signals. Some of the atmospheric effects can be corrected before the sensing data is subjected to further analysis and interpretation. These effects degrade the adequateness of received data. The new methods and supporting tools are developed to automate the measurement and consideration of the characteristics of the CC. It helps to build the information and mathematical models of nonlinear dynamic object such as the CC $[3,19$, 20], i.e. to solve the identification problem.

Building Volterra models and using them for visualization for such complex nature effects as waves of sea surface were well studied in [8-10]. This methodic allows building linear and nonlinear models for different systems. Modern continuous CCs are nonlinear stochastic inertial systems. The model in the form of integral Volterra series used to identify them $[3,4]$. The nonlinear and dynamic properties of such system are completely characterized by a sequence of multidimensional weighting functions - Volterra kernels). 


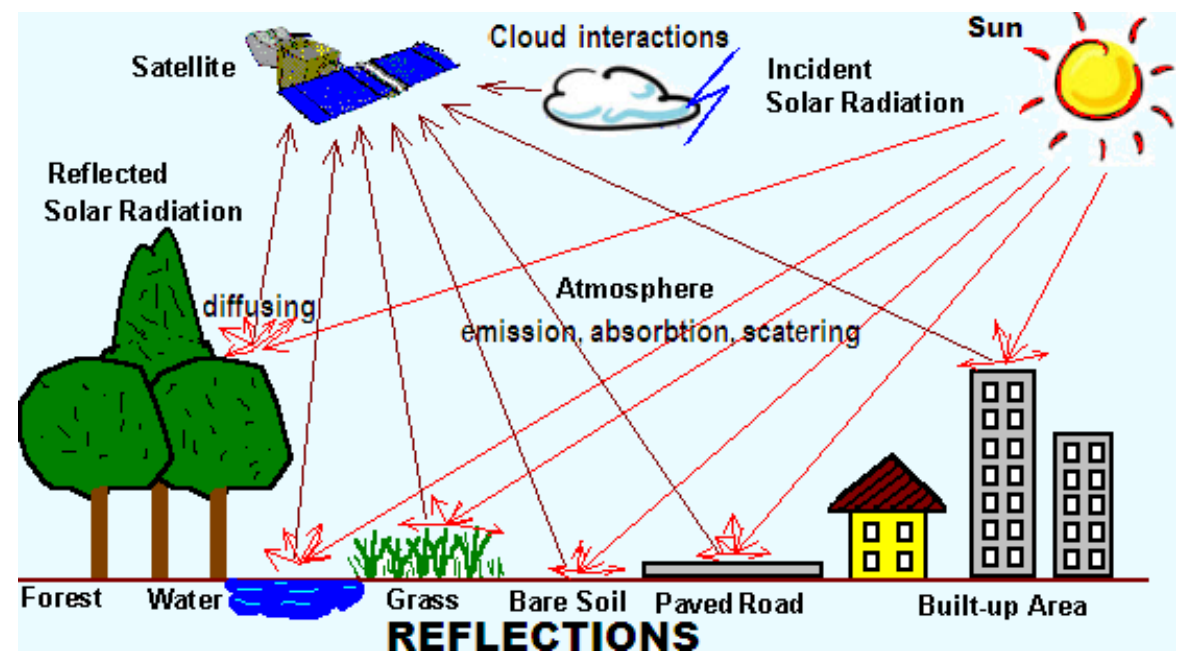

Figure 1. Environment effects in remote sensing systems

Building a model of nonlinear dynamic system in the form of a Volterra series lies in the choice of the test actions form. Also it uses the developed algorithm that allows determining the Volterra kernels and their Fourier-images for the measured responses (multidimensional amplitude-frequency characteristics (AFC) and phase-frequency characteristics (PFC)) to simulate the CC in the time or frequency domain, respectively [16-18, 23].

The additional research of new method of nonlinear dynamical systems identification, based on the Volterra model in the frequency domain is proposed. This method lies in $n$-fold differentiation of responses of the identifiable system by the amplitude of the test polyharmonic signals. The developed identification toolkit is used to build information model of the test nonlinear dynamic system in the form of the first, second and third order model. $[12,13,15,20,21]$

The aim of this work is to identify the continuous CC using Volterra model in the frequency domain, i.e. the determination of its multi-frequency characteristics on the basis of the data of the input-output experiment, using test polyharmonic signals and interpolation method to obtain model coefficients [14, 16-19, 21].

\section{Volterra models and identification of dynamical systems in the frequency domain}

Generally, "input-output" type ratio for nonlinear dynamical system can be presented by Volterra series [19]. 


$$
\begin{aligned}
& y[x(t)]=w_{0}(t)+\int_{0}^{\infty} w_{1}(\tau) x(t-\tau) d \tau+\int_{0}^{\infty} \int_{0}^{\infty} w_{2}\left(\tau_{1}, \tau_{2}\right) x\left(t-\tau_{1}\right) x\left(t-\tau_{2}\right) d \tau_{1} d \tau_{2}+ \\
& +\int_{0}^{\infty} \int_{0}^{\infty} \int_{0}^{\infty} w_{3}\left(\tau_{1}, \tau_{2}, \tau_{3}\right) x\left(t-\tau_{1}\right) x\left(t-\tau_{2}\right) x\left(t-\tau_{3}\right) d \tau_{1} d \tau_{2} d \tau_{3}+\ldots=w_{0}(t)+\sum_{n=1}^{\infty} y_{n}[x(t)]
\end{aligned}
$$

where the $n$-th partial component of response of the system is

$y_{n}[x(t)]=\int_{0}^{\infty} \ldots \int_{n \text { times }}^{\infty} \mathrm{w}_{n}\left(\tau_{1}, \ldots, \tau_{n}\right) \prod_{i=1}^{n} x\left(t-\tau_{i}\right) d \tau_{i}$

$x(t)$ and $y(t)$ are input and output signals of system respectively; $w_{n}\left(\tau_{1}, \tau_{2}, \ldots, \tau_{n}\right)$ - weight function or $n$-order Volterra kernel; $y_{n}[x(t)]-n$-th partial component of system's response; $w_{0}(t)$ - denotes free component of the series (for zero initial conditions $w_{0}(t)=0$ ); $t$-current time.

Commonly, the Volterra series are replaced by a polynomial, with only taking several first terms of series (1) into consideration. Nonlinear dynamical system identification in a form of Volterra series consists in $n$-dimensional weighting functions determination $w_{n}\left(\tau_{1}, \ldots, \tau_{n}\right)$ for time domain or it's Fourier transforms $W_{n}\left(j \omega_{1}, \ldots, j \omega_{n}\right)-n$-dimensional transfer functions for frequency domain.

Multidimensional Fourier transform for $n$-order Volterra kernel (1) is written in a form:

$W_{n}\left(j \omega_{1}, \ldots, j \omega_{n}\right)=F_{n}\left\langle w_{n}\left(\tau_{1}, \ldots, \tau_{n}\right)\right\rangle=\int_{0}^{\infty} \ldots \int_{0}^{\infty} w_{n}\left(\tau_{1}, \ldots, \tau_{n}\right) \exp \left(-j \sum_{i=1}^{n} \omega_{i} \tau_{i}\right) \prod_{i=1}^{n} d \tau_{i}$,

where $\left.F_{n} \backslash\right\rangle-n$-dimensional Fourier transform; $j=\sqrt{-1}$. Then the model of nonlinear system based on Volterra model in frequency domain can be represented as:

$y[x(t)]=\sum_{n=1}^{\infty} F_{n}^{-1}\left\langle W_{n}\left(j \omega_{1}, \ldots, j \omega_{n}\right) \prod_{i=1}^{n} X\left(j \omega_{i}\right)\right\rangle t_{1}=\ldots=t_{n}=t$,

where $F_{n}{ }^{-1}\langle\rangle$ - inverse $n$-dimensional Fourier transform; $X\left(j \omega_{i}\right)$ - Fourier transform of input signal.

Identification of nonlinear system in frequency domain consists in determination of absolute value and phase of multidimensional transfer function at given frequencies - multidimensional AFC $\left|W_{n}\left(j \omega_{1}, j \omega_{2}, \ldots, j \omega_{n}\right)\right|$ and PFC $\arg W_{n}\left(j \omega_{1}, j \omega_{2}, \ldots, j \omega_{n}\right)$ which are defined by formulas:

$$
\begin{gathered}
\left|W_{n}\left(j \omega_{1}, \ldots, j \omega_{n}\right)\right|=\sqrt{\left[\operatorname{Re}\left(W_{n}\left(j \omega_{1}, \ldots, j \omega_{n}\right)\right)\right]^{2}+\left[\operatorname{Im}\left(W_{n}\left(j \omega_{1}, \ldots, j \omega_{n}\right)\right)\right]^{2}}, \\
\quad \arg W_{n}\left(j \omega_{1}, \ldots, j \omega_{n}\right)=\operatorname{arctg} \frac{\operatorname{Im}\left[W_{n}\left(j \omega_{1}, \ldots, j \omega_{n}\right)\right]}{\operatorname{Re}\left[W_{n}\left(j \omega_{1}, \ldots, j \omega_{n}\right)\right]}
\end{gathered}
$$


where Re and Im - accordingly real and imaginary parts of a complex function of $n$ variables respectively.

An approximation method of identification of the nonlinear dynamical system based on Volterra series is offered [11, 14, 16-19]. During the identification of a Volterra kernel of $m$-th order significant effect on accuracy is rendered adjacent terms of a Volterra series. Therefore, it is necessary to apply the special methods, allowing minimizing this effect. The idea of such method lays in construction such expression of system responses to $N(1 \leq m \leq N)$ test input signals with the given amplitudes that with certain accuracy (accurate within to the thrown terms of order $N+1$ and above) would be equal to $m$-th term Volterra series:

$$
y_{m}[x(t)]=\sum_{j=1}^{N} c_{j} y\left[a_{j} x(t)\right]=\sum_{n=1}^{\infty}\left(\sum_{j=1}^{N} c_{j} a_{j}^{n}\right) \int_{0}^{\infty} \ldots . \int w_{n}\left(\tau_{1}, \ldots \tau_{n}\right) \prod_{i=1}^{n} x\left(t-\tau_{i}\right) d \tau_{i}
$$

where $a_{j}$ - amplitudes of test signals, random nonzero and pairwise different numbers; $c_{j}$ - real coefficients which are chosen in such way that in a right part of (4) all first $N$ terms are equal to 0 , except $m$-th, and the multiplier at a $m$-multiple integral became equal to 1 . This condition leads to a solution of the linear algebraic equations system concerning coefficients $c_{1}, \ldots, c_{N}$ :

$$
\begin{gathered}
c_{1} a_{1}+c_{2} a_{2}+\ldots+c_{N} a_{N}=0 \\
\ldots \\
c_{1} a_{1}^{m}+c_{2} a_{2}^{m}+\ldots+c_{N} a_{N}^{m}=1 \\
\ldots \\
c_{1} a_{1}^{N}+c_{2} a_{2}^{N}+\ldots+c_{N} a_{N}^{N}=0 .
\end{gathered}
$$

This system (5) always has a solution, and the unique one, as the system determinant differs from Vandermonde determinant with only a multiplier $a_{1} a_{2} \ldots a_{N}$. Thus, with any real numbers $a_{j}$, that different from zero and pairwise different, it is possible to find such numbers $c_{j}$ at which the linear combination (4) of system responses is equal to $m$-th term of a Volterra series accurate within to the thrown terms of series.

It is possible to build numberless assemblage of modes for expressions (4), by taking various numbers $a_{1}, \ldots, a_{N}$ and defining (5) coefficients $c_{1}, \ldots, c_{N}$ by them.

The choice of amplitudes $a_{j}$ should provide the convergence of series (1) and an minimum error $\Delta$ during extraction of a partial component $y_{m}[x(t)]$ according to (4) defined by reminder of series (1) - terms of degree $N+1$ and above

$$
\sum_{j=1}^{N} c_{j} y\left[a_{j} x(t)\right]=\int_{0}^{\infty} \ldots \int_{m}^{\infty} \int_{0}^{\infty} w_{m}\left(\tau_{1}, \ldots, \tau_{m}\right) \prod_{i=1}^{m} x\left(t-\tau_{i}\right) d \tau_{i}+\sum_{j=1}^{N} c_{j} \sum_{n=N+1}^{\infty} y_{n}\left[a_{j} x(t)\right]=y_{m}[x(t)]+\Delta
$$


If $x(t)$ - is a test effect with maximum admissible amplitude at which a series (1) converges, amplitudes $a_{j}$ should be no more than 1 by their absolute values: $\left|a_{j}\right| \leq 1$ for $\forall j=1,2, \ldots, N$.

The amplitudes of the test signals $a_{N j}^{(m)}$ and the corresponding coefficients $c_{N j}^{(m)}$ for responses are shown in table 1, where $m$ - order of the estimated Volterra kernel; $j$ - number of the experiment; $N$ - approximation order, i.e. quantity of identification experiments.

\begin{tabular}{|c|c|c|c|c|c|c|c|c|c|c|c|c|c|c|}
\hline$N$ & $m$ & \multicolumn{6}{|c|}{$a_{N j}^{(m)}$} & \multicolumn{6}{|c|}{$c_{N j}^{(m)}$} & $\Delta$ \\
\hline \multirow{2}{*}{2} & 1 & \multicolumn{3}{|c|}{-1} & \multicolumn{3}{|c|}{1} & \multicolumn{3}{|c|}{$-0,5$} & \multicolumn{3}{|c|}{0,5} & 1 \\
\hline & 2 & \multicolumn{3}{|c|}{-1} & \multicolumn{3}{|c|}{1} & \multicolumn{3}{|c|}{0,5} & \multicolumn{3}{|c|}{0,5} & 1 \\
\hline \multirow{3}{*}{4} & 1 & -1 & & 1 & $-0,5$ & &, 5 & 0,16 & & 0,167 & 1,333 & & ,333 & 3 \\
\hline & 2 & -1 & & 1 & $-0,644$ & & 544 & $-0,35$ & & $-0,354$ & 2,061 & & 061 & 4,8 \\
\hline & 3 & -1 & & 1 & 0,5 & &, 5 & $-0,66$ & & 0,667 & $-1,333$ & & ,333 & 4 \\
\hline \multirow{3}{*}{6} & 1 & $-0,8$ & 1 & -1 & $-0,3$ & 0,3 & 0,8 & 0,28 & 0,09 & $-0,09$ & $-2,13$ & 2,13 & 0,28 & 5 \\
\hline & 2 & 0,9 & -1 & 0,9 & 0,4 & $-0,4$ & 1 & $-0,8$ & 0,41 & $-0,8$ & 4,64 & 4,64 & 0,41 & 11,7 \\
\hline & 3 & 0,3 & 1 & 0,8 & $-0,8$ & -1 & $-0,3$ & $-5,46$ & $-1,11$ & 3,44 & $-3,44$ & 1,11 & 5,46 & 20 \\
\hline
\end{tabular}

Table 1. Numerical values of identification accuracy using approximation method

An interpolation method of identification of the nonlinear dynamical system based on Volterra series is offered $[12,13,15,20,21]$. It is used $n$-fold differentiation of a target signal on parameter-amplitude $a$ of test actions to separate the response of the nonlinear dynamical system on partial components $y_{n}[x(t)]$.

Affirmation 1. Let at input of system test signal of $a x(t)$ kind is given, where $x(t)$ - is arbitrary function and $a$-is scale coefficient (amplitude of signal), where $0<|a| \leq 1$, then for the selection of a partial component of the $n$-th order $\hat{y}_{n}(t)$ from measurement of the response nonlinear system $y[a x(t)]$ in the form of Volterra series, it is necessary to determine $\mathrm{n}$-th partial derivative of the total response amplitude a where $a=0$

$$
\hat{y}_{n}(t)=\int_{0}^{\infty} \ldots \int_{0}^{\infty} \mathrm{w}_{n}\left(\tau_{1}, \ldots, \tau_{n}\right) \prod_{i=1}^{n} x\left(t-\tau_{i}\right) d \tau_{i}=\left.\frac{1}{n !} \frac{\partial^{n} y[a x(t)]}{\partial a^{n}}\right|_{a=0}
$$

We use the method of extracting the partial components with the help of $n$-fold differentiation of the response $y[a x(t)]$ with respect to parameter-amplitude $a$ and the use of the derivative value at $a=0$.

Injecting an input signal $a x(t)$ where $a$ is the scaling factor (signal amplitude), one has the following response of the nonlinear system: 


$$
\begin{aligned}
& y[a \cdot x(t)]=a \int_{0}^{t} \mathrm{w}(\tau) \cdot x(t-\tau) d \tau+a^{2} \iint_{00}^{t t} \mathrm{w}_{2}\left(\tau_{1}, \tau_{2}\right) x\left(t-\tau_{1}\right) x\left(t-\tau_{2}\right) d \tau_{1} d \tau_{2}+ \\
& +a^{n} \int_{0}^{t} \ldots \int_{n t i m e} \mathrm{w}_{n}\left(\tau_{1}, \ldots, \tau_{n}\right) \prod_{r=1}^{n} x\left(t-\tau_{r}\right) d \tau_{r}+\ldots
\end{aligned}
$$

To distinguish the partial component of the $n$-th order, differentiate the system response $\mathrm{n}$ times with respect to the amplitude:

$$
\begin{gathered}
\frac{\partial^{n} y[a \cdot x(t)]}{\partial a^{n}}=n ! \int_{n}^{t} \ldots \int_{t \text { times }} \int_{0}^{t} \mathrm{w}_{n}\left(\tau_{1}, \ldots, \tau_{n}\right) \prod_{r=1}^{n} x\left(t-\tau_{r}\right) d \tau_{r}+ \\
(n+1) ! \cdot a \int_{0}^{t} \ldots \int_{\text {times }} \int_{0}^{t} \mathrm{w}_{n+1}\left(\tau_{1}, \ldots, \tau_{n+1}\right) \prod_{r=1}^{n+1} x\left(t-\tau_{r}\right) d \tau_{r}+\ldots
\end{gathered}
$$

Taking the value of the derivative at $a=0$, we finally obtain the expression for the partial component (7).

Formulas for numerical differentiation. Partial derivative should be substituted by form of finite difference for calculation. Differentiation of function, which was set in discrete points, could be accomplished by means of numerical computing after preliminary smoothing of measured results. Various formulas for the numerical differentiation are known, which differ from each other by means of error.

Let's use universal reception which allows to substitute a derivative of any $n$ order for differential ratio so that the error from such replacement for function $y(a)$ was any beforehand set order of $p$ approximation concerning a step of $h=\Delta a$ of computational mesh on amplitude. Method of undetermined coefficient for equality

$$
\frac{d^{n} y(a)}{d a^{n}}=\frac{1}{h^{n}} \sum_{r=-r_{1}}^{r_{2}} c_{r} y(a+r h)+O\left(h^{p}\right),
$$

where the coefficients $c_{r}$ are taken not depending on $h$, $r=-r_{1},-r_{1}+1, \ldots,-1,0,1, \ldots, r_{2}-1, r_{2}$, so that equality (10) was fair. Limits of summation $r_{1} \geq 0$ and $r_{2} \geq 0$ could be arbitrary, but so that the differential relation $h^{-n} \sum c_{r} y(a+r h)$ of $r_{1}+r_{2}$ order have to satisfy to inequality $r_{1}+r_{2} \geq n+p-1$.

To define the $c_{r}$ it is necessary to solve the following set of equations 


$$
\left[\begin{array}{cccc}
1 & 1 & \ldots & 1 \\
-r_{1} & -r_{1}+1 & \ldots & r_{2} \\
\ldots & \ldots & \ldots & \ldots \\
\left(-r_{1}\right)^{n-1} & \left(-r_{1}+1\right)^{n-1} & \ldots & r_{2}{ }^{n-1} \\
\left(-r_{1}\right)^{n} & \left(-r_{1}+1\right)^{n} & \ldots & r_{2}{ }^{n} \\
\left(-r_{1}\right)^{n+1} & \left(-r_{1}+1\right)^{n+1} & \ldots & r_{2}{ }^{n+1} \\
\ldots & \ldots & \ldots & \ldots \\
\left(-r_{1}\right)^{n+p-1} & \left(-r_{1}+1\right)^{n+p-1} & \ldots & r_{2}{ }^{n+p-1}
\end{array}\right] \cdot\left[\begin{array}{c}
c_{-r_{1}} \\
c_{-r_{1}+1} \\
\ldots \\
c_{-1} \\
c_{0} \\
c_{1} \\
\ldots \\
c_{r_{2}}
\end{array}\right]=\left[\begin{array}{c}
0 \\
0 \\
\ldots \\
0 \\
n ! \\
0 \\
\ldots \\
0
\end{array}\right]
$$

If $r_{1}+r_{2}=n+p-1$, then inscribed in $n+p$ equality forms linear system concerning the same number of $c_{r}$ unknown. The determiner of this system is Vandermonde's determiner and differs from zero. Thus, there is only one set of $n$ coefficients, satisfying the system.

If $r_{1}+r_{2} \geq n+p$, then there are many such sets of coefficients $c_{r}$.

On the basis of (10) in [20] the formulas of derivative calculation of the first, second and third orders are received at $\mathrm{a}=0$ with use of the central differences for equidistant nodes of the computational grid.

In work formulas for numerical differentiation with use of the central differences for equidistant assembly are used. Volterra kernel of the first order is determined by formulas as the first derivative at $r_{1}=r_{2}=1, r_{1}=r_{2}=2$ or $r_{1}=r_{2}=3$ respectively

$$
\begin{aligned}
& y_{0}^{\prime}=\frac{1}{2 h}\left(-y_{-1}+y_{1}\right) \\
& y_{0}^{\prime}=\frac{1}{12 h}\left(y_{-2}-8 y_{-1}+8 y_{1}-y_{2}\right), \\
& y_{0}^{\prime}=\frac{1}{60 h}\left(-y_{-3}+9 y_{-2}-45 y_{-1}+45 y_{1}-9 y_{2}+y_{3}\right) .
\end{aligned}
$$

Volterra kernel of the first order is determined by formulas as the first derivative at $r_{1}=r_{2}=1$, $r_{1}=r_{2}=2$ or $r_{1}=r_{2}=3$ respectively

$$
\begin{aligned}
& y_{0}^{\prime \prime}=\frac{1}{h^{2}}\left(y_{-1}-2 y_{0}+y_{1}\right) \\
& y_{0}^{\prime \prime}=\frac{1}{12 h^{2}}\left(-y_{-2}+16 y_{-1}-30 y_{0}+16 y_{1}-y_{2}\right) \\
& y_{0}^{\prime \prime}=\frac{1}{180 h^{2}}\left(2 y_{-3}-27 y_{-2}+270 y_{-1}-490 y_{0}+270 y_{1}-27 y_{2}+2 y_{3}\right) .
\end{aligned}
$$


Volterra kernel of the first order is determined by formulas as the first derivative at $r_{1}=r_{2}=2$ or $r_{1}=r_{2}=3$ respectively

$$
\begin{aligned}
& y_{0}^{\prime \prime \prime}=\frac{1}{2 h^{3}}\left(-y_{-2}+2 y_{-1}-2 y_{1}+y_{2}\right), \\
& y_{0}^{\prime \prime \prime}=\frac{1}{8 h^{3}}\left(y_{-3}-8 y_{-2}+13 y_{-1}-13 y_{1}+8 y_{2}-y_{3}\right) .
\end{aligned}
$$

In the formulas written above, we use the following notations

$y_{0}^{\prime}=y^{\prime}(0), y_{0}^{\prime \prime}=y^{\prime \prime}(0), y^{\prime \prime \prime}=y^{\prime \prime \prime}(0) ; y_{r}=y(r h), r=0, \pm 1, \pm 2 ; \pm 3$

where we put $y_{0}=0$, since identification nonlinear systems is implemented with zero initial conditions. The test polyharmonic effects for identification in the frequency domain representing by signals of such type:

$$
x(t)=\sum_{k=1}^{n} A_{k} \cos \left(\omega_{k} t+\varphi_{k}\right),
$$

where $n$-the order of transfer function being estimated; $A_{k}, \omega_{k}$ and $\varphi_{k}$-accordingly amplitude, frequency and a phase of $k$-th harmonics. In research, it is supposed every amplitude of $A_{k}$ to be equal, and phases $\varphi_{k}$ equal to zero.

For identification in the frequency domain the test polyharmonic signals are used. We prove: Statement. If test polyharmonic signal is used in form

$$
x(t)=A \sum_{k=1}^{n} \cos \omega_{k} t=\frac{A}{2} \sum_{k=1}^{n}\left(e^{j \omega_{k} t}+e^{-j \omega_{k} t}\right)
$$

then the $n$-th partial component of the response of test system can be written in form:

$$
\begin{aligned}
& y_{n}(t)=\frac{A^{n}}{2^{n-1}} \sum_{m=0}^{E(n / 2)} C_{n}^{m} \sum_{k_{1}=1}^{n} \ldots \sum_{k_{n}=1}^{n}\left|W_{n}\left(-j \omega_{k_{1^{\prime}}} \ldots-j \omega_{k_{m^{\prime}}} j \omega_{k_{m+1}} \ldots j \omega_{k_{n}}\right)\right| \times \\
& \times \cos \left(\left(-\sum_{l=0}^{m} \omega_{k_{l}}+\sum_{l=m+1}^{n} \omega_{k_{l}}\right) t+\arg W_{n}\left(-j \omega_{k_{1^{\prime}}} \ldots-j \omega_{k_{m^{\prime}}} j \omega_{k_{m+1}} \ldots j \omega_{k_{n}}\right)\right),
\end{aligned}
$$

where $E(n / 2)$ - function used to obtain the of integer part of the value.

The component with frequency $\omega_{1}+\ldots+\omega_{n}$ is extracted from the response to test signal (15): 


$$
A^{n}\left|W_{n}\left(j \omega_{1}, \ldots, j \omega_{n}\right)\right| \cos \left[\left(\omega_{1}+\ldots+\omega_{n}\right) t+\arg W_{n}\left(j \omega_{1}, \ldots, j \omega_{n}\right)\right] .
$$

Certain limitations should be imposed while choosing of frequency polyharmonic test signals in a process determining multidimensional AFC and PFC. This is the reason why the values of AFC and PFC in this unallowable points of multidimensional frequency space can be calculated using interpolation only. In practical realization of nonlinear dynamical systems identification it is needed to minimize number of such undefined points at the range of multidimensional frequency characteristics determination. This was performed to provide a minimum of restrictions on choice of frequency of the test signal. It is shown that existed limitation can be weakened. New limitations on choice of frequency are reducing number of undefined points.

After analyzing the (18) it is defined: to obtain Volterra kernels for nonlinear dynamical system in frequency domain the limitations on choice of frequencies of test polyharmonic signals have to be restricted. These restrictions provide inequality of combination frequencies in the test signal harmonics. The theorem about choice of test signals frequencies is proven.

The theorem about choice of test signals frequencies. For the definite filtering of a response of the harmonics with combination frequencies $\omega_{1}+\omega_{2}+\ldots+\omega_{n}$ within the $n$-th partial component it is necessary and sufficient to keep the frequency from being equal to another combination frequencies of type $k_{1} \omega_{1}+\ldots+k_{n} \omega_{n}$, where the coefficients $\left\{k_{i} \mid i=1,2, \ldots, n\right\}$ must satisfy the conditions:

- number $K$ of negative value coefficients $\left(k_{i}<0\right)$ is in $0 \leq K \leq E(n / 2)$ (where $E$ - function used to obtain the of integer part of the value);

$$
\begin{aligned}
& -\sum_{i=1}^{n}\left|k_{i}\right| \leq n ; \\
& \text { - } \sum_{i=1}^{n}\left|k_{i}\right| \equiv n(\bmod 2), n-\sum_{i=1}^{n}\left|k_{i}\right|=2 l, l \in N .
\end{aligned}
$$

It was shown that during determination of multidimensional transfer functions of nonlinear systems it is necessary to consider the imposed constraints on choice of the test polyharmonic signal frequencies. This provides inequality of combination frequencies in output signal harmonics: $\omega_{1} \neq 0, \omega_{2} \neq 0$ and $\omega_{1} \neq \omega_{2}$ for the second order identification procedure, and $\omega_{1} \neq 0, \omega_{2} \neq 0, \omega_{3} \neq 0, \omega_{1} \neq \omega_{2}, \omega_{1} \neq \omega_{3}, \omega_{2} \neq \omega_{3}, 2 \omega_{1} \neq \omega_{2}+\omega_{3}, 2 \omega_{2} \neq \omega_{1}+\omega_{3}, 2 \omega_{3} \neq \omega_{1}+\omega_{2}, 2 \omega_{1} \neq \omega_{2}-$ $\omega_{3}, 2 \omega_{2} \neq \omega_{1}-\omega_{3}, 2 \omega_{3} \neq \omega_{1}-\omega_{2}, 2 \omega_{1} \neq-\omega_{2}+\omega_{3}, 2 \omega_{2} \neq-\omega_{1}+\omega_{3}$ and $2 \omega_{3} \neq-\omega_{1}+\omega_{2}$ for the third order identification procedure.

The amplitudes of the test signals $a_{i}^{(n)}$ and the corresponding coefficients $c_{i}^{(n)}$ for responses are shown in Table 2, where $n$ - order of the estimated Volterra kernel; $i$-number of the experiment $(i=1,2, \ldots, N)$, where $N=r_{1}+r_{2}$, i.e. number of interpolation knots (number of experiments). 


\begin{tabular}{|c|c|c|c|c|c|c|c|c|c|c|c|c|c|}
\hline$n$ & $N$ & $a_{1}^{(n)}$ & $a_{2}^{(n)}$ & $a_{3}^{(n)}$ & $a_{4}^{(n)}$ & $a_{5}^{(n)}$ & $a_{6}^{(n)}$ & $c_{1}^{(n)}$ & $c_{2}^{(n)}$ & $c_{3}^{(n)}$ & $C_{4}{ }^{(n)}$ & $c_{5}^{(n)}$ & $C_{6}^{(n)}$ \\
\hline \multirow{3}{*}{1} & 2 & -1 & 1 & & & & & $-0,5$ & 0,5 & & & & \\
\hline & 4 & -1 & $-0,5$ & 0,5 & 1 & & & 0,0833 & $-0,6667$ & 0,6667 & $-0,0833$ & & \\
\hline & 6 & -1 & -0.67 & $-0,33$ & 0,33 & 0,67 & 1 & $-0,0167$ & 0,15 & $-0,75$ & 0,75 & $-0,15$ & 0,0167 \\
\hline \multirow{3}{*}{2} & 2 & -1 & 1 & & & & & 1 & 1 & & & & \\
\hline & 4 & -1 & $-0,5$ & 0,5 & 1 & & & $-0,0833$ & 1,3333 & 1,3333 & $-0,0833$ & & \\
\hline & 6 & -1 & -0.67 & $-0,33$ & 0,33 & 0,67 & 1 & 0,0111 & $-0,15$ & 1,5 & 1,5 & $-0,15$ & 0,0111 \\
\hline \multirow{2}{*}{3} & 4 & -1 & $-0,5$ & 0,5 & 1 & & & $-0,5$ & 1 & -1 & 0,5 & & \\
\hline & 6 & -1 & -0.67 & $-0,33$ & 0,33 & 0,67 & 1 & 0,125 & -1 & 1,625 & $-1,625$ & 1 & $-0,125$ \\
\hline
\end{tabular}

Table 2. Amplitudes and corresponding coefficients of the interpolation method

\section{The techniques of test system identification}

Described method was tested using nonlinear test system (fig. 2) represented by Riccati equation

$$
\frac{d y(t)}{d t}+\alpha y(t)+\beta y^{2}(t)=u(t)
$$

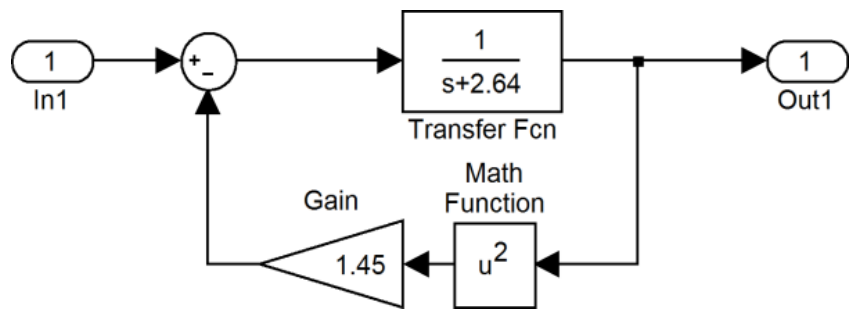

Figure 2. Simulink-model of the test system 
Analytical expressions of AFC and PFC for the first, second and third order model were received:

$$
\begin{aligned}
& \left|W_{1}(j \omega)\right|=\frac{1}{\sqrt{\alpha^{2}+\omega^{2}}}, \arg W_{1}(j \omega)=-\operatorname{arctg} \frac{\omega}{\alpha} ; \\
& \left|W_{2}\left(j \omega_{1}, j \omega_{2}\right)\right|=\frac{\beta}{\sqrt{\left(\alpha^{2}+\omega_{1}^{2}\right)\left(\alpha^{2}+\omega_{2}^{2}\right)\left[\alpha^{2}+\left(\omega_{1}+\omega_{2}\right)^{2}\right]}}, \\
& \arg W_{2}\left(j \omega_{1}, j \omega_{2}\right)=-\operatorname{arctg} \frac{\left(2 \alpha^{2}-\omega_{1} \omega_{2}\right)\left(\omega_{1}+\omega_{2}\right)}{\alpha\left(\alpha^{2}-\omega_{1} \omega_{2}\right)-\alpha\left(\omega_{1}+\omega_{2}\right)^{2}} ; \\
& \left|W_{3}\left(j \omega_{1}, j \omega_{2}, j \omega_{3}\right)\right|=\sqrt{\left[\operatorname{Re}\left(W_{3}\left(j \omega_{1}, j \omega_{2}, j \omega_{3}\right)\right)\right]^{2}+\left[\operatorname{Im}\left(W_{3}\left(j \omega_{1}, j \omega_{2}, j \omega_{3}\right)\right)\right]^{2}}= \\
& =\frac{2 \beta^{2}}{3} \frac{1}{\sqrt{\left[\alpha^{2}+\left(\omega_{1}+\omega_{2}+\omega_{3}\right)^{2}\right]\left(\alpha^{2}+\omega_{1}^{2}\right)\left(\alpha^{2}+\omega_{2}^{2}\right)\left(\alpha^{2}+\omega_{3}^{2}\right)}} \times \\
& \times \frac{\sqrt{\left.\left[3 \alpha^{2}-\left(\omega_{1}+\omega_{3}\right)\left(\omega_{1}+\omega_{2}\right)-\left(\omega_{1}+\omega_{2}\right)\right]\left(\omega_{2}+\omega_{3}\right)-\left(\omega_{1}+\omega_{3}\right)\left(\omega_{2}+\omega_{3}\right)\right]^{2}+16 \alpha^{2}\left(\omega_{1}+\omega_{2}+\omega_{3}\right)^{2}}}{\sqrt{\left[\alpha^{2}+\left(\omega_{1}+\omega_{2}\right)^{2}\right]\left[\alpha^{2}+\left(\omega_{1}+\omega_{3}\right)^{2}\right]\left[\alpha^{2}+\left(\omega_{2}+\omega_{3}\right)^{2}\right]}} \\
& \arg W_{3}\left(j \omega_{1}, j \omega_{2}, j \omega_{3}\right)=\operatorname{arctg} \frac{\operatorname{Im} W_{3}\left(j \omega_{1}, j \omega_{2}, j \omega_{3}\right)}{\operatorname{Re} W_{3}\left(j \omega_{1}, j \omega_{2}, j \omega_{3}\right)}=-\operatorname{arctg} \frac{D A-C B}{A B+C D},
\end{aligned}
$$

where

$$
\begin{aligned}
& u=\alpha^{3}-\alpha \omega_{1} \omega_{3}-\alpha \omega_{2} \omega_{3}-\alpha\left(\omega_{1}+\omega_{2}+\omega_{3}\right) ; \\
& v=\left(\omega_{1}+\omega_{2}+\omega_{3}\right)\left(2 \alpha^{2}-\omega_{1} \omega_{3}-\omega_{2} \omega_{3}\right) ; \\
& w=\left(\alpha^{2}-\omega_{1} \omega_{2}-\omega_{2} \omega_{3}\right)\left(\alpha^{2}-\omega_{1} \omega_{2}-\omega_{1} \omega_{3}\right)-\alpha^{2}\left(\omega_{1}+\omega_{2}+\omega_{3}\right)^{2} ; \\
& x=\alpha\left(\omega_{1}+\omega_{2}+\omega_{3}\right)\left(2 \alpha^{2}-2 \omega_{1} \omega_{2}-\omega_{1} \omega_{3}-\omega_{2} \omega_{3}\right) .
\end{aligned}
$$

The main purpose was to identify the multi-frequency performances characterizing nonlinear and dynamical properties of nonlinear test system [11-21]. Volterra model in the form of the $1^{\text {st }}, 2^{\text {nd }}$ and $3^{\text {rd }}$ order polynomial is used. Thus, test system properties are characterized by transfer functions of $W_{1}(j \omega), W_{2}\left(j \omega_{1}, j \omega_{2}\right), W_{3}\left(j \omega_{1}, j \omega_{2}, j \omega_{3}\right)$ - by Fourier-images of weight functions $w_{1}(t), w_{2}\left(t_{1}, t_{2}\right)$ and $w_{3}\left(t_{1}, t_{2}, t_{3}\right)$.

Structure charts of identification procedure - determinations of the $1^{\text {th }}, 2^{\text {nd }}$ and $3^{\text {rd }}$ order AFC of CC are presented accordingly in figs. 3-5.

The weighted sum is formed from received signals - responses of each group from figs. 3-5. As a result the partial components of CC responses $y_{1}(t), y_{2}(t)$ and $y_{3}(t)$ are got. For each partial component of response the Fourier transform (the FFT is used) is calculated, and from received spectrum only an informative harmonics (which amplitudes represent values of required characteristics of the first, second and third orders AFC) are taken. 


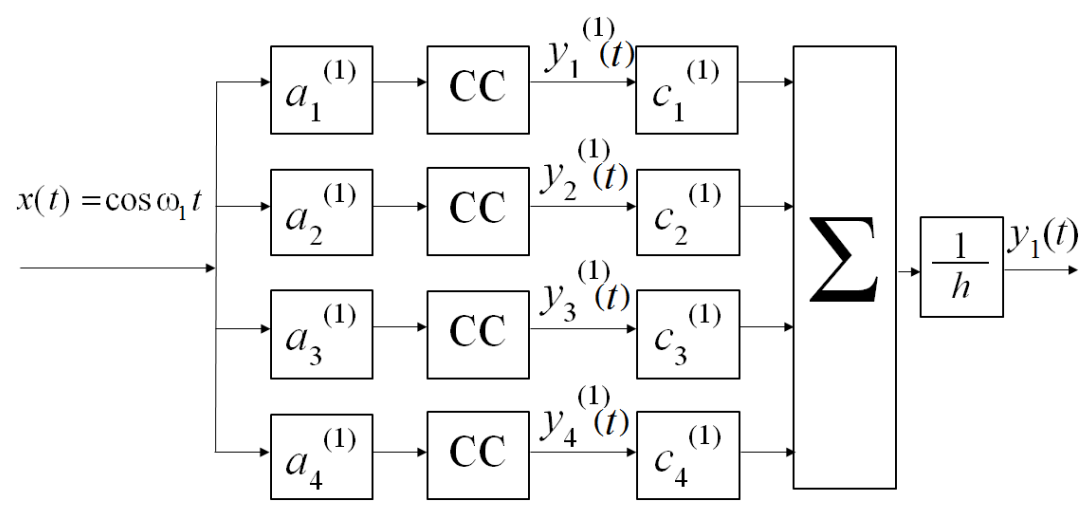

Figure 3. The structure chart of identification using $1^{\text {st }}$ order Volterra model in frequency domain, number of experiments $N=4: a_{1}=-2 h, a_{2}=-h, a_{3}=h, a_{4}=2 h ; c_{1}=-1 / 12, c_{2}=-2 / 3, c_{3}=2 / 3, c_{4}=1 / 12$

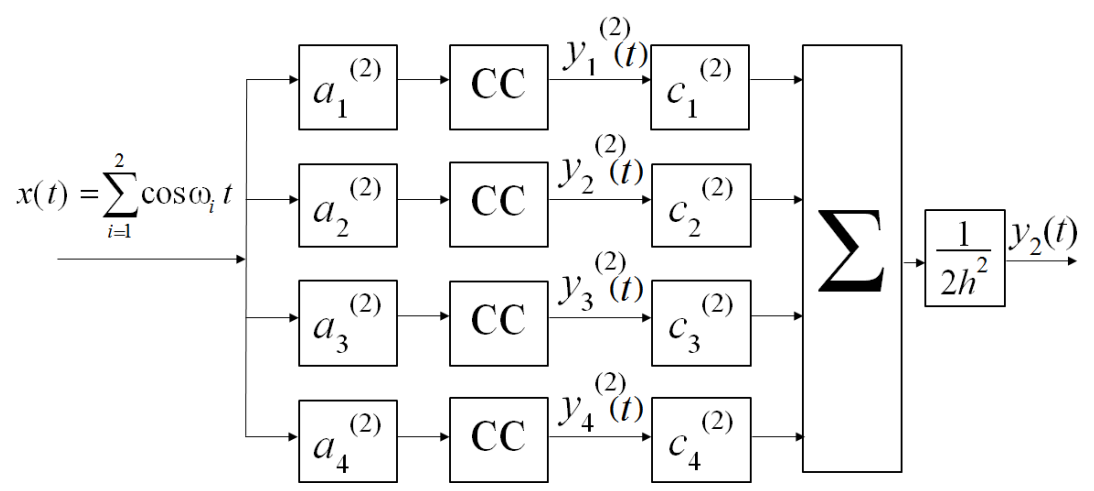

Figure 4. The structure chart of identification using $2^{\text {nd }}$ order Volterra model in frequency domain, number of experiments $N=4: a_{1}=-2 h, a_{2}=-h, a_{3}=h, a_{4}=2 h ; c_{1}=-1 / 12, c_{2}=4 / 3, c_{3}=4 / 3, c_{4}=-1 / 12$

The first order AFC $\left|W_{1}(j \omega)\right|$ and PFC $\arg W_{1}(j \omega)$ is received by extracting the harmonics with frequency $\omega$ from the spectrum of the CC partial response $y_{1}(t)$ to the test signal $x(t)=(A / 2) \cos \omega t$.

The second order AFC I $W_{2}\left(j \omega, j\left(\omega+\Omega_{1}\right)\right) \mid$ and PFC $\arg W_{2}\left(j \omega, j\left(\omega+\Omega_{1}\right)\right)$ having $\omega_{1}=\omega$ and $\omega_{2}=\omega$ $+\Omega_{1}$ were received by extracting the harmonics with summary frequency $\omega_{1}+\omega_{2}$ from the spectrum of the CC partial response $y_{2}(t)$ to the test signal $x(t)=(A / 2)\left(\cos \omega_{1} t+\cos \omega_{2} t\right)$.

The third order AFC I $W_{3}\left(j \omega, j\left(\omega+\Omega_{1}\right), j\left(\omega+\Omega_{2}\right)\right) \mid$ and PFC $\arg W_{3}\left(j \omega, j\left(\omega+\Omega_{1}\right), j\left(\omega+\Omega_{2}\right)\right)$ having $\omega_{1}=\omega, \omega_{2}=\omega+\Omega_{1}$ and $\omega_{3}=\omega+\Omega_{2}$, were received by extracting the harmonics with summary frequency $\omega_{1}+\omega_{2}+\omega_{3}$ from the spectrum of the CC partial response $y_{3}(t)$ to the test signal $x(t)=(A / 2)\left(\cos \omega_{1} t+\cos \omega_{2} t+\cos \omega_{3} t\right)$.

The results (first, second and third order AFC and PFC which had been received after procedure of identification) are represented in figs. 6-8. 


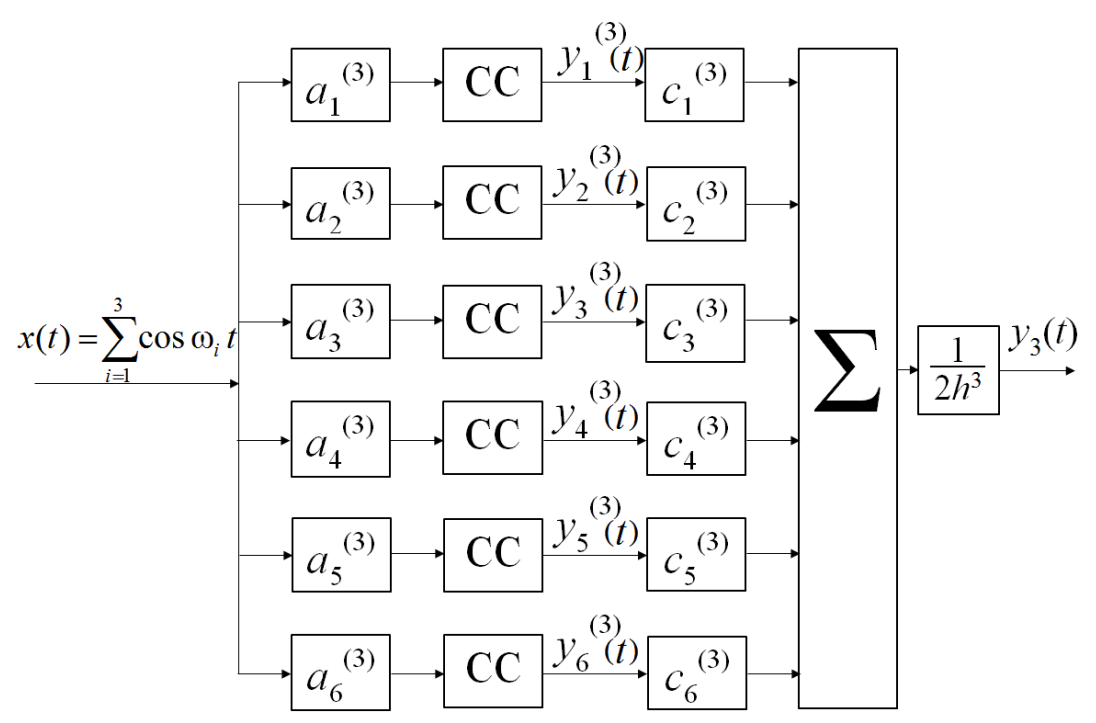

Figure 5. The structure chart of identification using $3^{\text {rd }}$ order Volterra model in frequency domain, number of experiments $N=6$ : $a_{1}=-3 h, a_{2}=-2 h, a_{3}=-h, a_{4}=h, a_{5}=2 h, a_{6}=3 h ; c_{1}=-1 / 8, c_{2}=-1, c_{3}=13 / 8, c_{4}=-13 / 8, c_{5}=1, c_{6}=1 / 8$
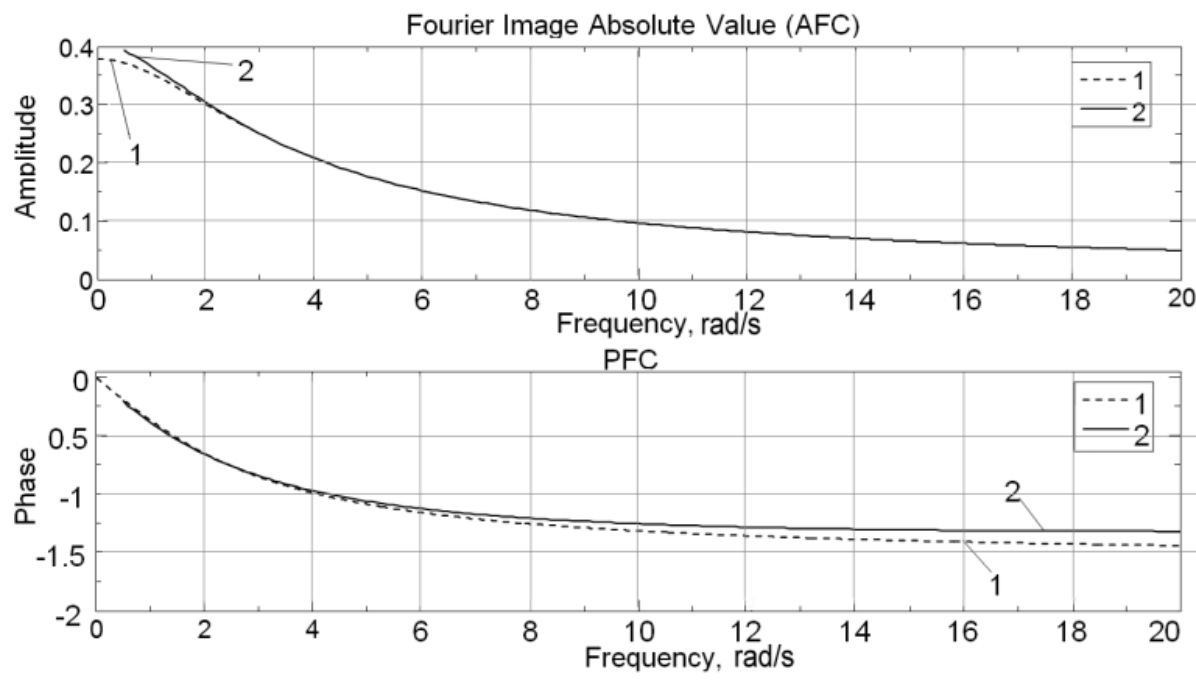

Figure 6. First order AFC and PFC of the test system: analytically calculated values (1), section estimation values with number of experiments for the model $N=4$ (2) 


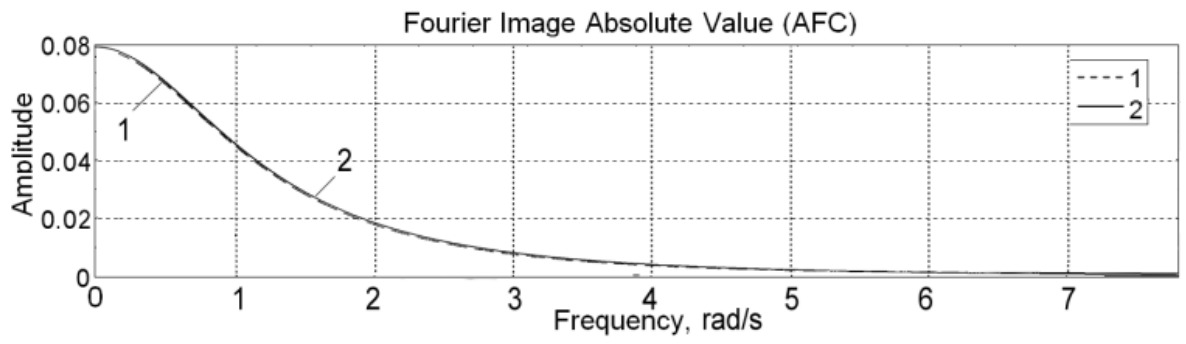

PFC

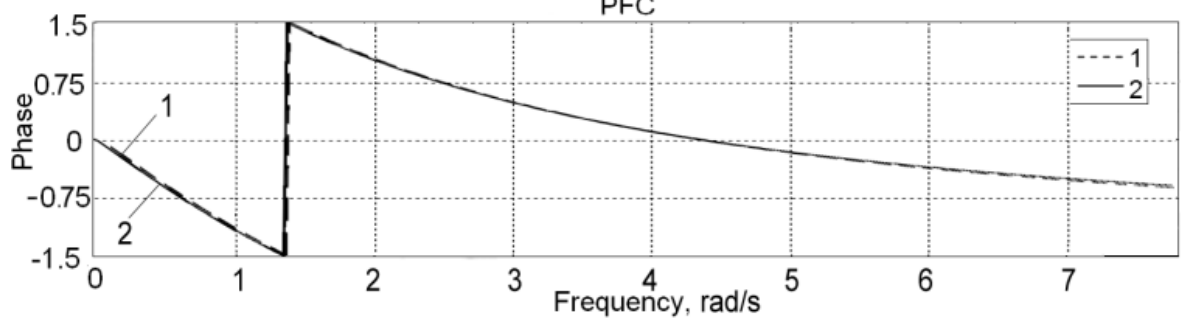

Figure 7. Second order AFC and PFC of the test system: analytically calculated values (1), sub-diagonal cross-section values with number of experiments for the model $N=4(2), \Omega_{1}=0,01 \mathrm{rad} / \mathrm{s}$
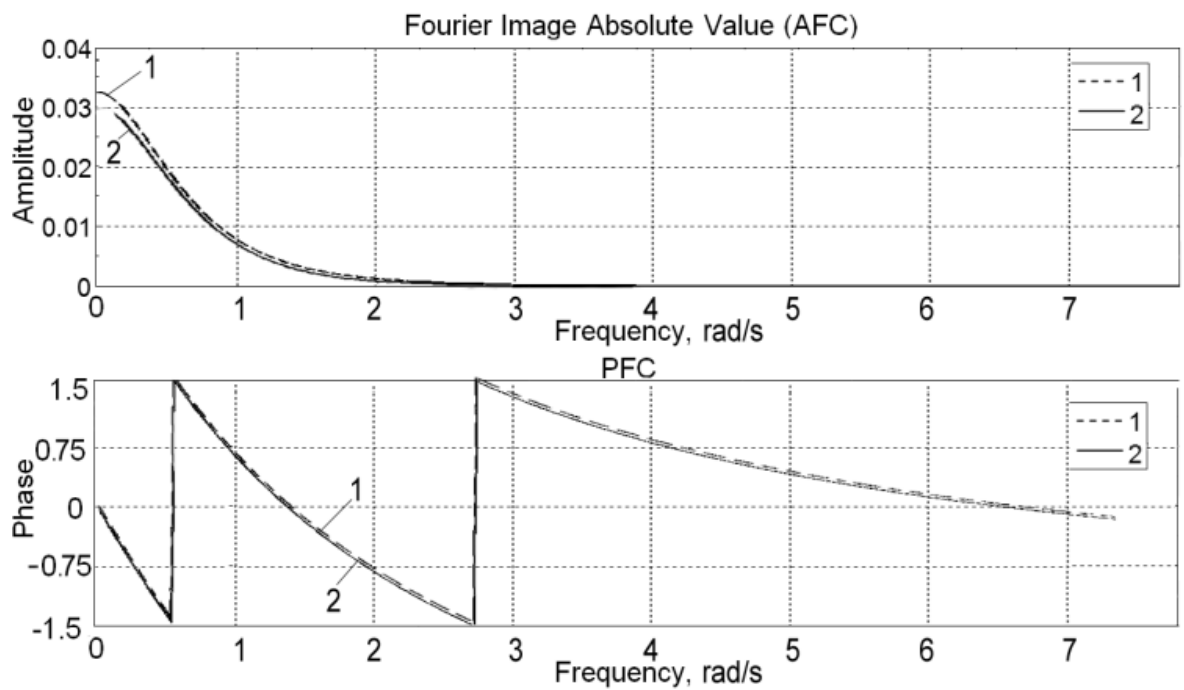

Figure 8. Third order AFC and PFC of the test system: analytically calculated values (1), sub-diagonal cross-section values with number of experiments for the model $N=6(2), \Omega_{1}=0,01 \mathrm{rad} / \mathrm{s}, \Omega_{2}=0,1 \mathrm{rad} / \mathrm{s}$

The second and third order surfaces for AFC and PFC received after procedure of the test system identification are shown in fig. 9 and fig. 10 respectively. 

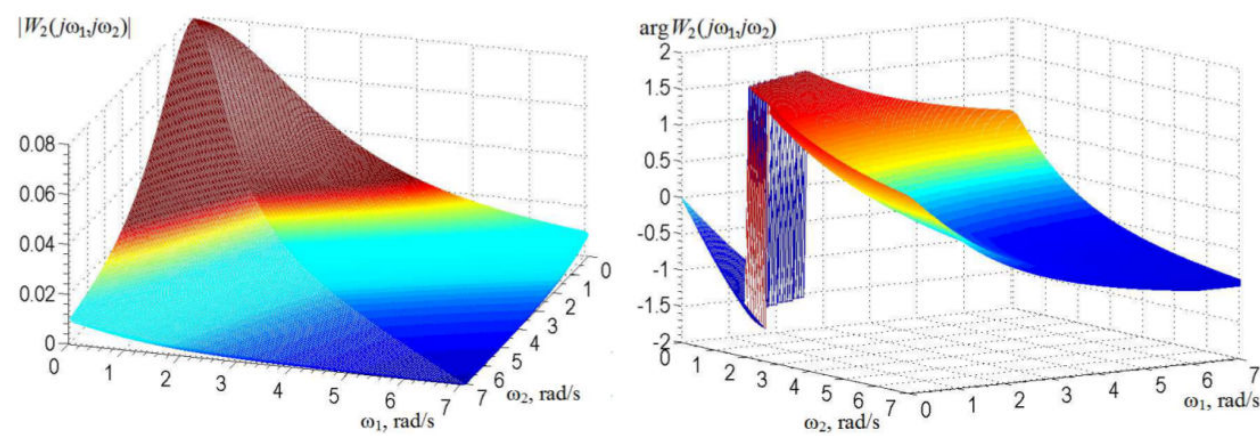

Figure 9. Surface of the test system AFC (left) and PFC (right) built of the second order subdiagonal cross-sections received for $N=4$
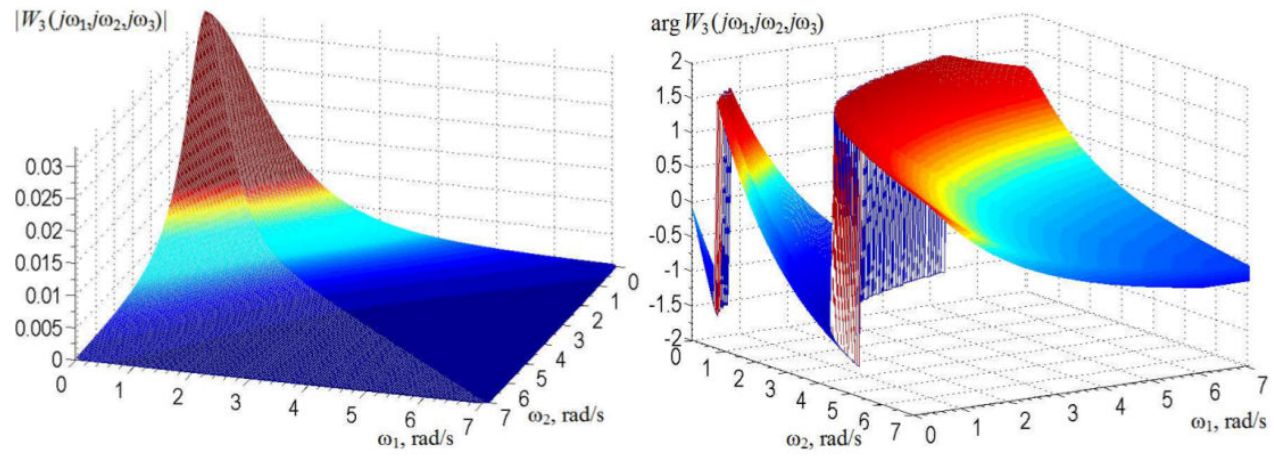

Figure 10. Surface of the test system AFC (left) and PFC (right) built of the third order subdiagonal cross-sections received for $N=6, \omega_{3}=0,1 \mathrm{rad} / \mathrm{s}$

Presented surfaces are built from sub-diagonal cross-sections which were received separately. $\Omega_{1}$ was used as growing parameter of identification with different value for each cross-section in second order characteristics. Fixed value of $\Omega_{2}$ and growing value of $\Omega_{1}$ were used as parameters of identification to obtain different value for each cross-section in third order characteristics.

Numerical values of identification accuracy using interpolation method for the test system are represented in table 3, where: $n$ - order of the estimated Volterra kernel, $N$-approximation order/number of interpolation knots (number of experiments). 


\begin{tabular}{|c|c|c|c|}
\hline$n$ & $N$ & AFC relative error, $\%$ & PFC relative error, $\%$ \\
\hline \multirow{3}{*}{1} & 2 & 2.1359 & 2.5420 \\
\hline & 4 & 0.3468 & 2.0618 \\
\hline & 6 & 0.2957 & 1.9311 \\
\hline \multirow{3}{*}{2} & 2 & 30.2842 & 76.8221 \\
\hline & 4 & 2.0452 & 3.7603 \\
\hline & 6 & 89.2099 & 5.9438 \\
\hline \multirow{2}{*}{3} & 4 & 10.981 & 1.628 \\
\hline & 6 & 10.7642 & 1.5522 \\
\hline
\end{tabular}

Table 3. Numerical values of identification accuracy using interpolation method

Comparison of the numerical values for identification accuracy using interpolation method [17-18] and approximation one [13-16] for the test system is presented in table 4.

\begin{tabular}{|c|c|c|c|c|c|}
\hline \multirow{2}{*}{$n$} & \multirow{2}{*}{$N$} & \multicolumn{2}{|c|}{ AFC relative error, $\%$} & \multicolumn{2}{|c|}{ PFC relative error, \% } \\
\hline & & approximation & interpolation & approximation & interpolation \\
\hline \multirow{3}{*}{1} & 2 & 3.6429 & 2.1359 & 3.3451 & 2.5420 \\
\hline & 4 & 1.1086 & 0.3468 & 3.1531 & 2.0618 \\
\hline & 6 & 0.8679 & 0.2957 & 3.1032 & 1.9311 \\
\hline \multirow{3}{*}{2} & 2 & 26.0092 & 30.2842 & 30.2842 & 76.8221 \\
\hline & 4 & 3.4447 & 2.0452 & 2.0452 & 3.7603 \\
\hline & 6 & 7.3030 & 89.2099 & 4.6408 & 5.9438 \\
\hline \multirow{2}{*}{3} & 4 & 72.4950 & 10.981 & 10.9810 & 1.628 \\
\hline & 6 & 74.4204 & 10.7642 & 10.7642 & 1.5522 \\
\hline
\end{tabular}

Table 4. Identification accuracy using approximation and interpolation methods

\section{The study of noise immunity of the identification method}

Experimental researches of the noise immunity of the identification method were made. The main purpose was the studying of the noise impact (noise means the inexactness of the 
measurements) to the characteristics of the test system model using interpolation method in frequency domain.

The first step was the measurement of the level of useful signal (harmonic cosine test signal shown in fig. 11a) after test system (Out2 in fig. 12). The amplitude of this signal was defined as the $100 \%$ of the signal power.

After that procedure the Random Noise signal (with the form shown in fig. 11b) where added to the test system output signal. This steps where performed to simulate inexactness of the measurements in the model. The sum of these two signals for the linear test model signal is shown in fig. 13.

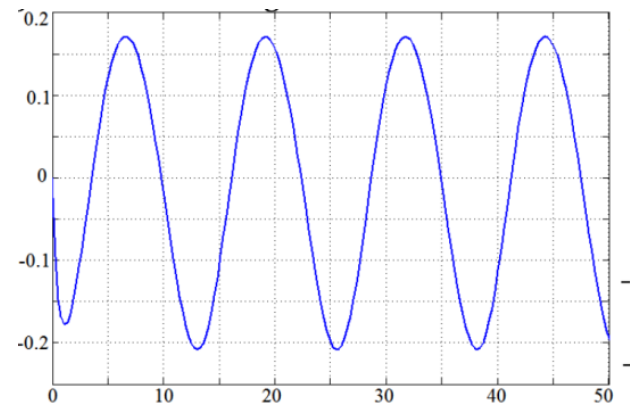

a)

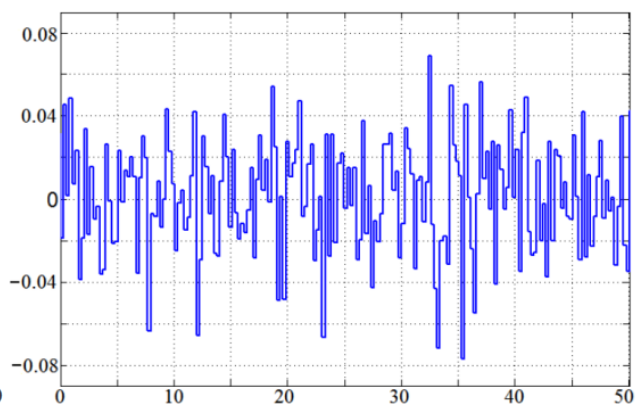

b)

Figure 11. a) Test signal and b) random noise with $50 \%$ amplitude of test signal

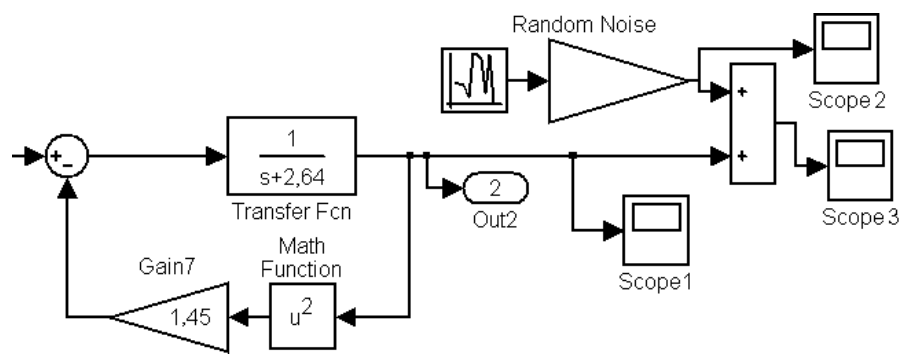

Figure 12. The Simulink model of the test system with noise generator and osillosopes

The simulations with the test model were performed. Different noise levels were defined for different order of the Volterra model.

The adaptive wavelet denoising was used to reduce the noise impact on final characteristics of the test system. The Daubechie wavelet of the 2 and 3 level was chosen (fig. 14) and used for the AFC and PFC denoising respectively $[2,5,7]$. 


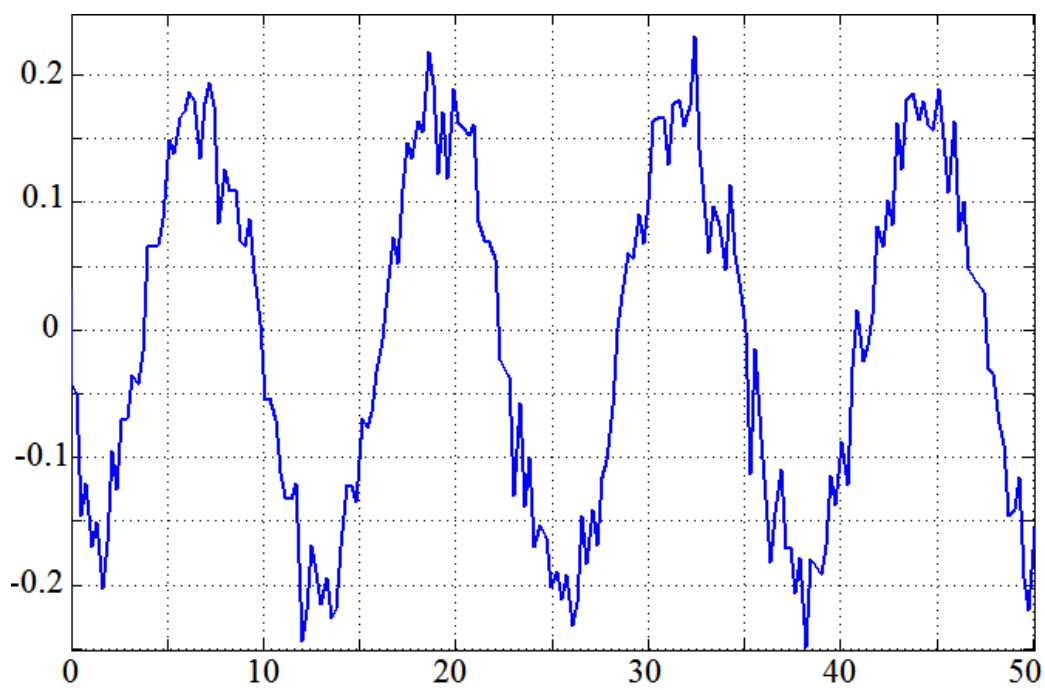

Figure 13. The "noised" signal of the test system, level of noise is $50 \%$ of source signal

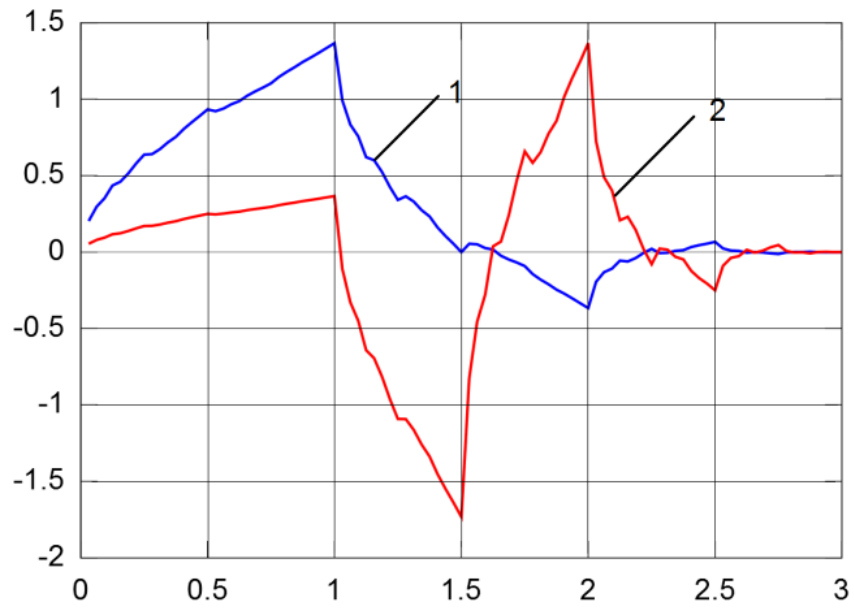

Figure 14. The Daubechies $2^{\text {nd }}$ level scaling (1) and wavelet (2) functions

The first order (linear) model was tested with the level of noise $50 \%$ and $10 \%$ and showed excellent level of noise immunity. The noised (fig. 15a) and de-noised (filtered) (fig. 15b) characteristics (AFC and PFC) with level of noise $50 \%$ are presented.

The second order (nonlinear) model was tested with the level of noise $10 \%$ and $1 \%$ and showed good level of noise immunity. The noised (fig. 16a) and de-noised (filtered) (fig. 16b) characteristics (AFC and PFC) with level of noise 10\% are presented. 

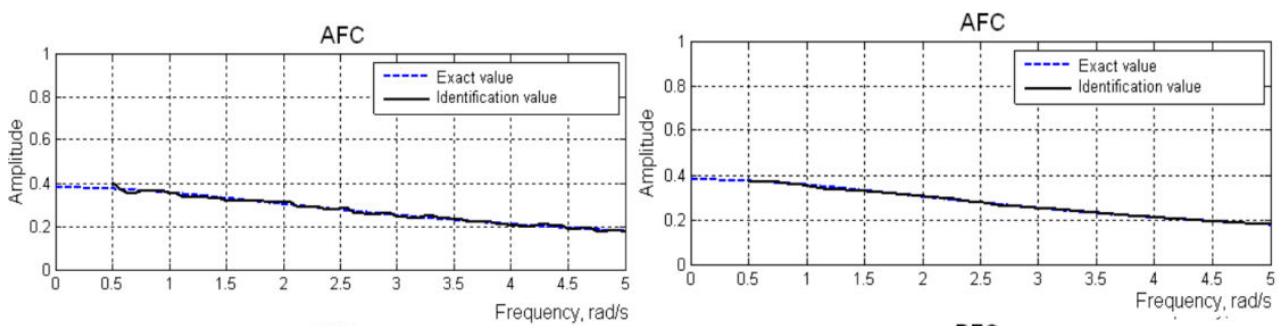

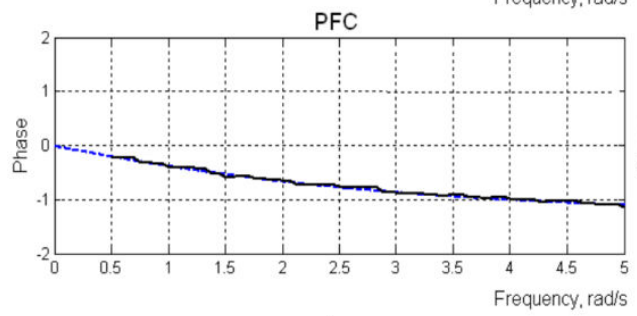

a)

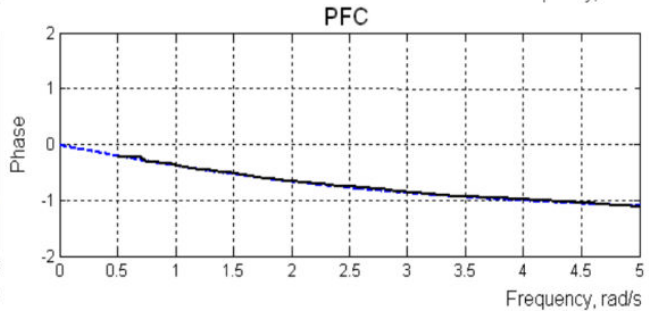

b)

Figure 15. Noised (a) and denoised (b) characteristics (AFC - top, PFC - bottom) of the $1^{\text {st }}$ order model of the test system with level of noise $50 \%$
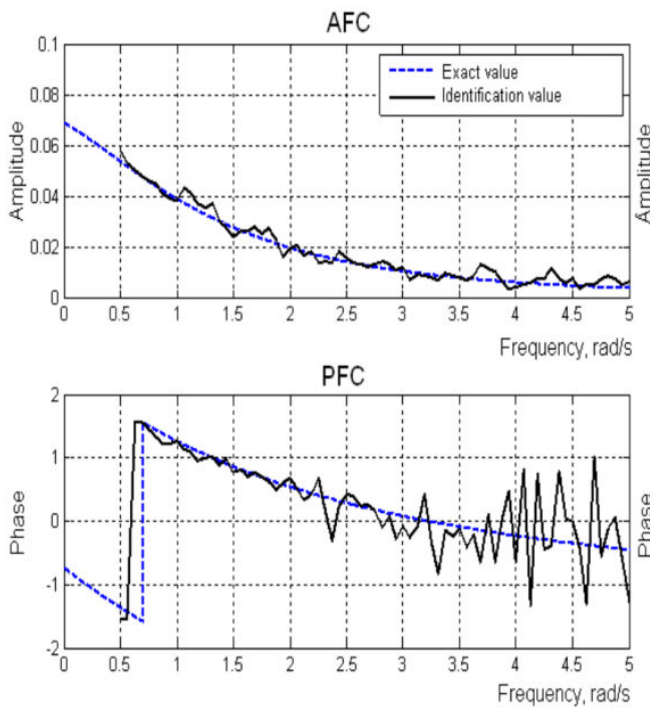

a)

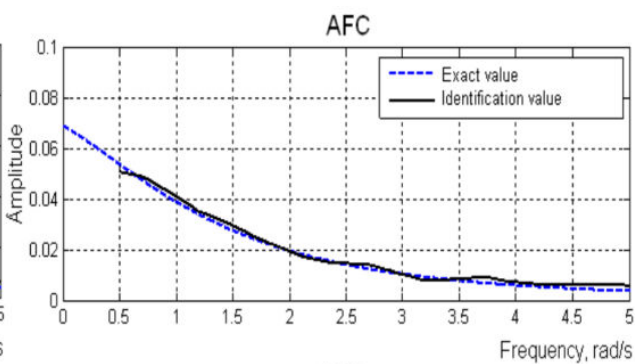

PFC

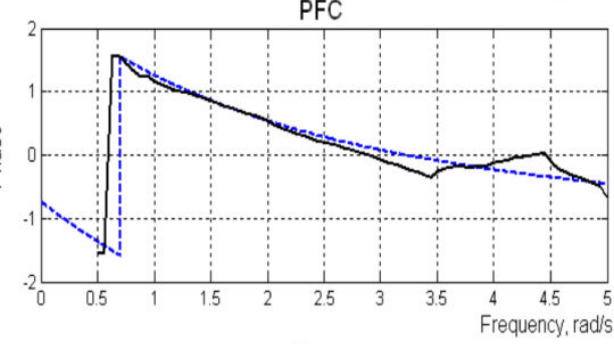

b)

Figure 16. Noised (a) and denoised (b) characteristics (AFC - top, PFC - bottom) of the $2^{\text {nd }}$ order model of the test system with level of noise $10 \%$ 
The third order (nonlinear) model was tested with the level of noise $10 \%$ and $1 \%$ and showed good level of noise immunity. The noised (fig. 17a) and de-noised (filtered) (fig. 17b) characteristics (AFC and PFC) with level of noise $1 \%$ are presented.
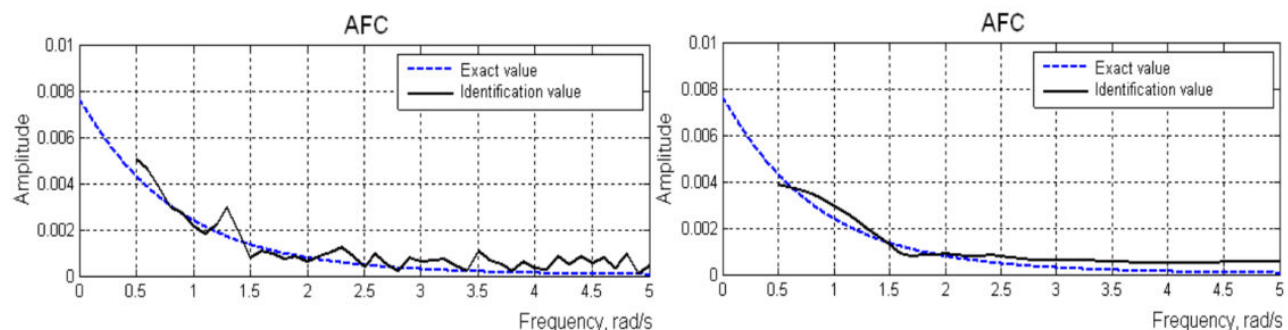

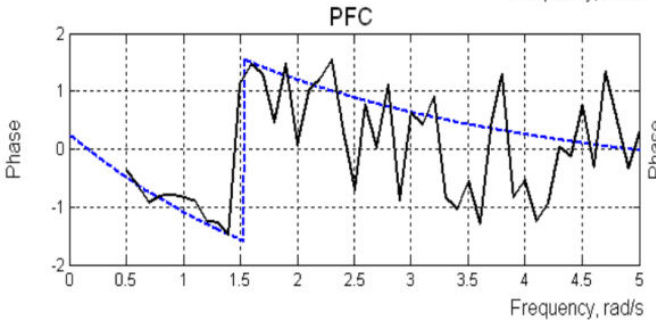

a)

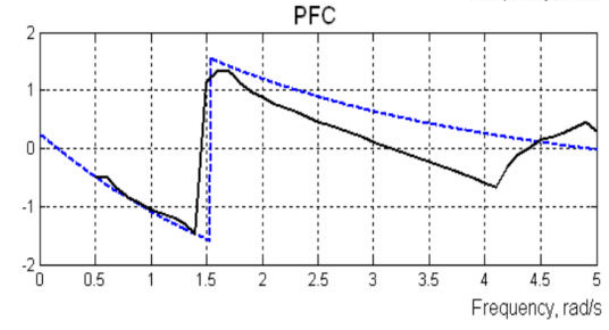

b)

Figure 17. Noised (a) and denoised (b) characteristics (AFC - top, PFC - bottom) of the $3^{\text {rd }}$ order model of the test system with level of noise $1 \%$

The numerical values of standard deviation (SD) of the identification accuracy before and after wavelet denoising procedure are presented in Table 5.

\begin{tabular}{|c|c|c|c|c|c|c|c|}
\hline \multirow[b]{2}{*}{$n$} & \multirow[b]{2}{*}{$N$} & \multicolumn{2}{|c|}{ Noise level $=10 \%$} & \multicolumn{2}{|c|}{ Noise level = 1\% } & \multicolumn{2}{|c|}{ Improvement } \\
\hline & & SD for AFC & SD for PFC & SD for AFC & SD for PFC & $\begin{array}{c}\text { for AFC, } \\
\text { times }\end{array}$ & for PFC, times \\
\hline \multicolumn{8}{|c|}{ (without / with denoising) } \\
\hline \multirow{3}{*}{1} & 2 & $\begin{array}{c}0.000097 / \\
0.000063\end{array}$ & $\begin{array}{c}0.09031 / \\
0.07541\end{array}$ & - & - & 1,540 & 1,198 \\
\hline & 4 & $\begin{array}{c}0.000271 / \\
0.000181\end{array}$ & $\begin{array}{c}0.07804 / \\
0.06433\end{array}$ & - & - & 1,497 & 1,213 \\
\hline & 6 & $\begin{array}{c}0.000312 / \\
0.000223\end{array}$ & $\begin{array}{c}0.12913 / \\
0.09889\end{array}$ & - & - & 1,399 & 1,306 \\
\hline 2 & 2 & $\begin{array}{c}0.000920 / \\
0.000670\end{array}$ & $\begin{array}{c}0.52063 / \\
0.51465\end{array}$ & - & - & 1,373 & 1,012 \\
\hline
\end{tabular}




\begin{tabular}{|c|c|c|c|c|c|c|c|}
\hline \multirow[b]{2}{*}{$n$} & \multirow[b]{2}{*}{$N$} & \multicolumn{2}{|c|}{ Noise level = $10 \%$} & \multicolumn{2}{|c|}{ Noise level = 1\% } & \multicolumn{2}{|c|}{ Improvement } \\
\hline & & SD for AFC & SD for PFC & SD for AFC & SD for PFC & $\begin{array}{c}\text { for AFC, } \\
\text { times }\end{array}$ & for PFC, times \\
\hline & 4 & $\begin{array}{c}0.001972 / \\
0.001663\end{array}$ & $\begin{array}{c}0.28004 / \\
0.06877\end{array}$ & - & - & 1,186 & 4,072 \\
\hline & 6 & $\begin{array}{c}0.004165 / \\
0.003908\end{array}$ & $\begin{array}{c}0.39260 / \\
0.19237\end{array}$ & - & - & 1,066 & 2,041 \\
\hline \multirow{2}{*}{3} & 4 & - & - & $\begin{array}{c}0.000288 / \\
0.000288\end{array}$ & $\begin{array}{c}0.89857 / \\
0.61251\end{array}$ & 1,003 & 1,467 \\
\hline & 6 & - & - & $\begin{array}{c}0.000461 / \\
0.000352\end{array}$ & $\begin{array}{c}0.84868 / \\
0.59319\end{array}$ & 1,310 & 1,431 \\
\hline
\end{tabular}

Table 5. Standard deviation for interpolation method with noise impact (bold font shows the best values)

The diagrams showing the improvement of standard deviation for identification accuracy using the adaptive wavelet denoising of the received characteristics (AFC and PFC) are shown in fig. 18 and fig. 19 respectively.

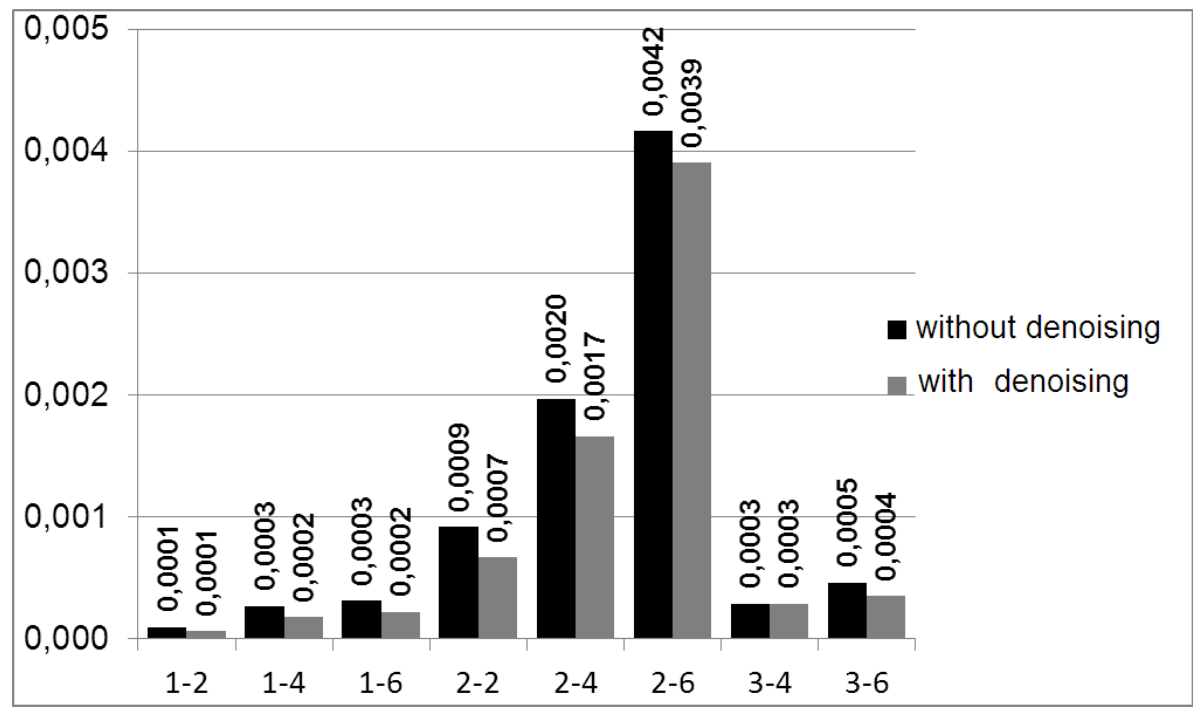

Figure 18. Standard deviation changing for AFC using adaptive Wavelet-denoising 


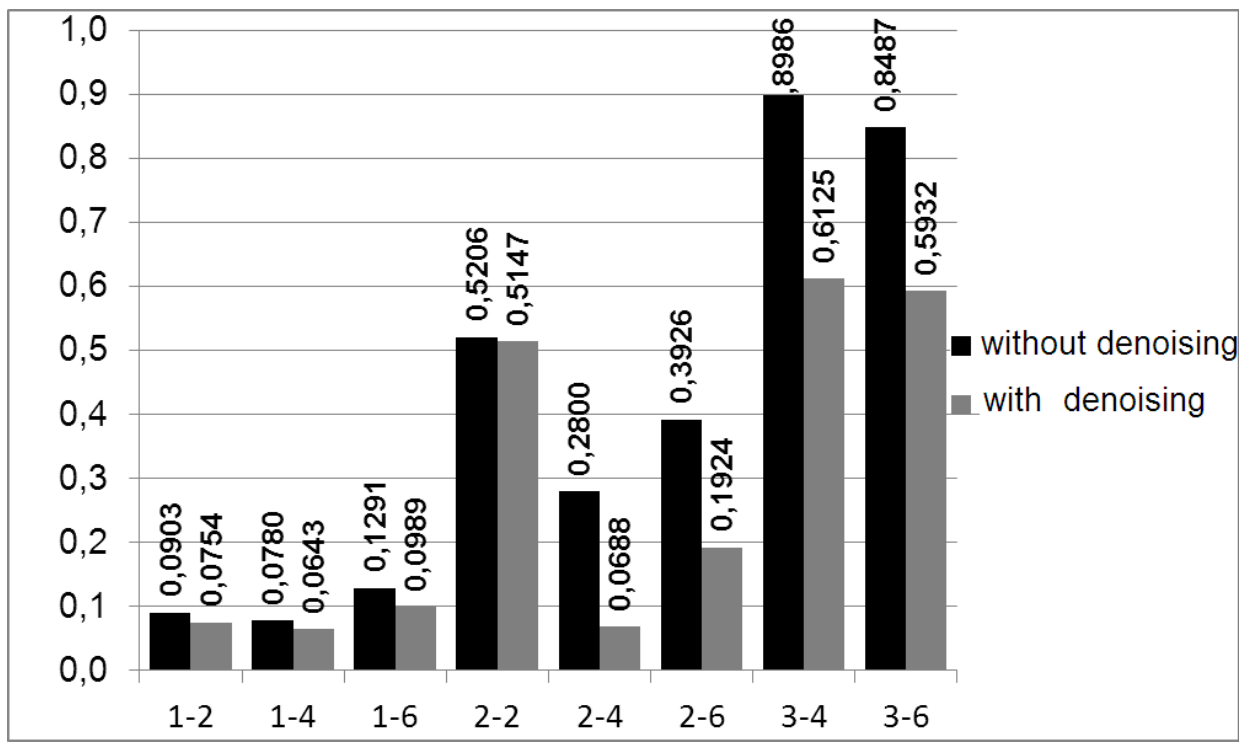

Figure 19. Standard deviation changing for PFC using adaptive Wavelet-denoising

\section{The technique and hardware-software tools of radiofrequency CC identification}

Experimental research of the Ultra High Frequency range CC were done. The main purpose was the identification of multi-frequency characteristics that characterize nonlinear and dynamical properties of the CC. Volterra model in the form of the second order polynomial is used. Thus physical CC properties are characterized by transfer functions of $W_{1}(j 2 \pi f)$, $W_{2}\left(j 2 \pi f_{1}, j 2 \pi f_{2}\right)$ and $W_{3}\left(j 2 \pi f_{1}, j 2 \pi f_{2}, j 2 \pi f_{3}\right)$ - by the Fourier-images of weighting functions $w_{1}(t)$, $w_{2}\left(t_{1}, t_{2}\right)$ and $w_{3}\left(t_{1}, t_{2}, t_{3}\right)$.

Implementation of identification method on the IBM PC computer basis has been carried out using the developed software in Matlab software. The software allows automating the process of the test signals forming with the given parameters (amplitudes and frequencies). Also this software allows transmitting and receiving signals through an output and input section of PC soundcard, to produce segmentation of a file with the responses to the fragments, corresponding to the CC responses being researched on test polyharmonic effects with different amplitudes.

In experimental research two identical marine transceivers S.P.RADIO A/S SAILOR RT2048 VHF (the range of operational frequencies is $154,4-163,75 \mathrm{MHz}$ ) and IBM PC with Creative Audigy 4 soundcards were used. Sequentially AFC of the first and second orders were defined. The method of identification with number of experiments $N=4$ was applied. Structure charts 
of identification procedure-determinations of the $1^{\text {st }}, 2^{\text {nd }}$ and $3^{\text {rd }}$ order AFC of CC are presented accordingly in figs. 3-5. The general scheme of a hardware-software complex of the CC identification, based on the data of input-output type experiment is presented in fig. 20.

The CC received responses $y\left[a_{i} x(t)\right]$ to the test signals $a_{i} x(t)$, compose a group of the signals, which amount is equal to the used number of experiments $N(N=4)$, shown in fig. 21. In each following group the signals frequency increases by magnitude of chosen step. A crosscorrelation was used to define the beginning of each received response.

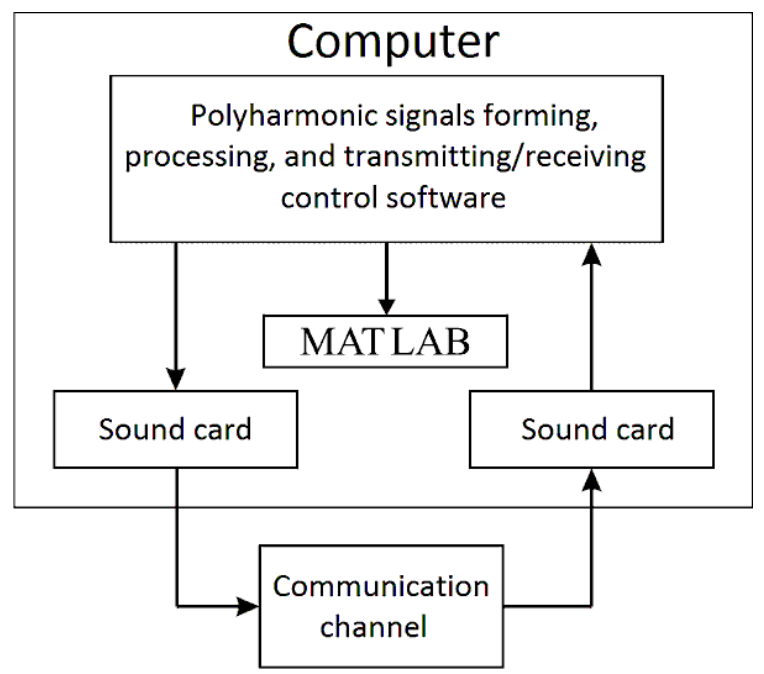

Figure 20. The general scheme of the experiment

Maximum allowed amplitude in described experiment with use of sound card was $A=0,25 \mathrm{~V}$ (defined experimentally). The range of frequencies was defined by the sound card pass band $(20 \ldots 20000 \mathrm{~Hz})$, and frequencies of the test signals has been chosen from this range, taking into account restrictions specified above. Such parameters were chosen for the experiment: start frequency $f_{\mathrm{s}}=125 \mathrm{~Hz}$; final frequency $f_{\mathrm{e}}=3125 \mathrm{~Hz}$; a frequency change step $F=125 \mathrm{~Hz}$; to define AFC of the second order determination, an offset on frequency $F_{1}=f_{2}-f_{1}$ was increasingly growing from 201 to $3401 \mathrm{~Hz}$ with step $100 \mathrm{~Hz}$.

The weighed sum is formed from received signals - responses of each group (figs. 3-5). As a result we get partial components of the response of the CC $y_{1}(t)$ and $y_{2}(t)$. For each partial component of the response a Fourier transform (the Fast Fourier Transform is used) is calculated. Only informative harmonics (which amplitudes represents values of required characteristics of the first, second and third order AFC) are taken from received spectrum.

The first order amplitude-frequency characteristic $\left|W_{1}(j 2 \pi f)\right|$ is received by extracting the harmonics with frequency f from the spectrum of the partial response of the CC $y_{1}(t)$ to the test signal $x(t)=(A / 2) \cos 2 \pi f t$. 


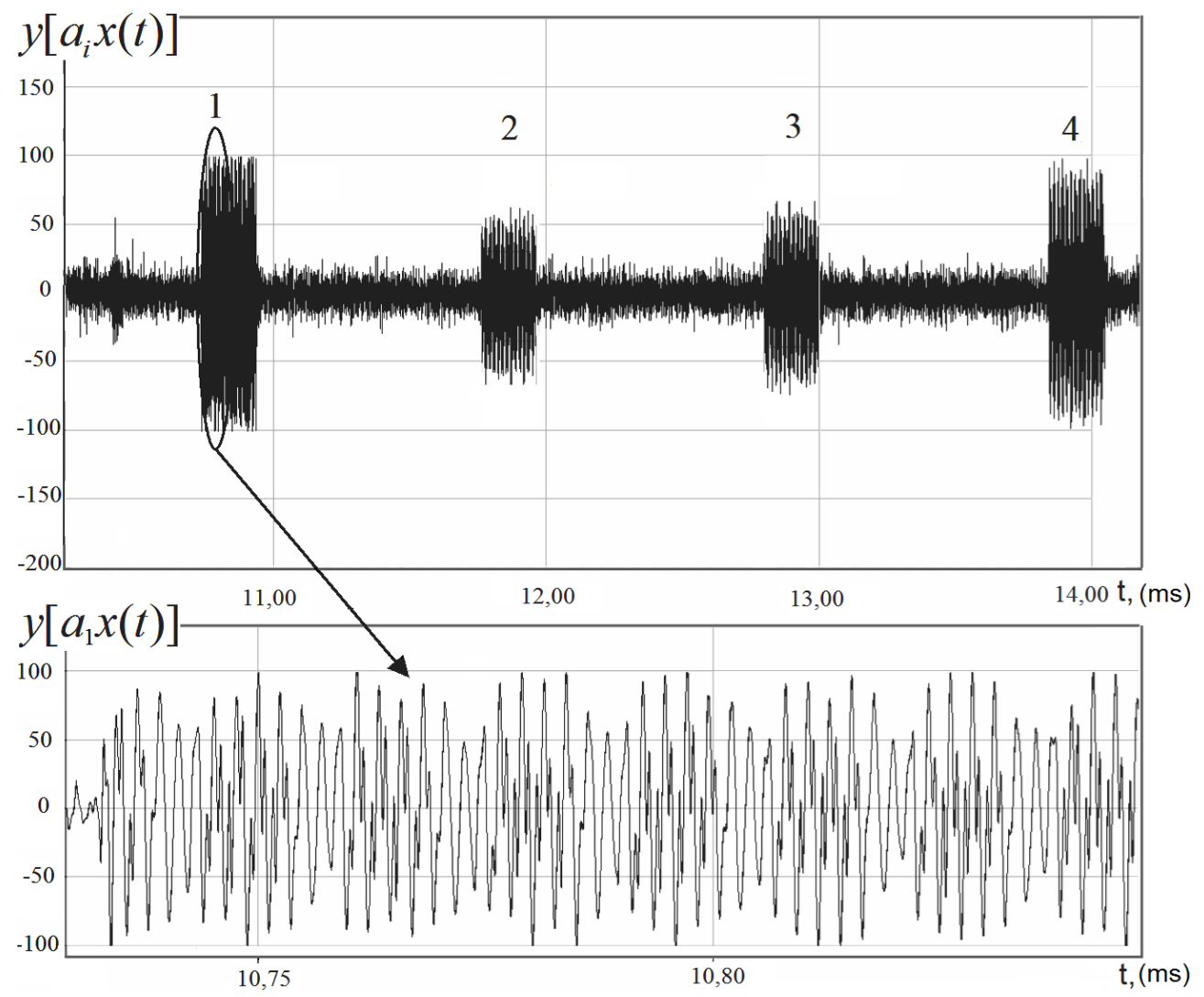

Figure 21. The group of signals received from CC with amplitudes:-1 (1);-1/2 (2); $1 / 2(3) ; 1(4) ; N=4$

The second order AFC $\left|W_{2}\left(j 2 \pi f_{1}, j 2 \pi f_{2}\right)\right|$, where $f_{1}=f$ and $f_{2}=f+F_{1}$ was received by extracting the harmonics with summary frequency $f_{1}+f_{2}$ from the spectrum of the partial response of the CC $y_{2}(t)$ to the test signal $x(t)=(A / 2)\left(\cos 2 \pi f_{1} t+\cos 2 \pi f_{2} t\right)$.

The third order AFC $\left|W_{3}\left(j 2 \pi f_{1}, j 2 \pi f_{2}, j 2 \pi f_{3}\right)\right|$, where $f_{1}=f_{,} f_{2}=f+F_{1}$ and $f_{3}=127,5 \mathrm{~Hz}$ were received by extracting the harmonics with summary frequency $f_{1}+f_{2}+f_{3}$ from the spectrum of the partial response of the CC $y_{3}(t)$ to the test signal $x(t)=(A / 2)\left(\cos 2 \pi f_{1} t+\cos 2 \pi f_{2} t+\cos 2 \pi f_{3} t\right)$.

The wavelet noise-suppression was used to smooth the output data of the experiment [9]. The results received after digital data processing of the data of experiments (wavelet "Coiflet" denoising) for the first, second and third order AFC are presented in fig. 22-25.

The surfaces shown in fig. 24-25 are built from sub-diagonal sections that are received separately. We used $F_{1}$ as growing parameter of identification with different value for each section. 


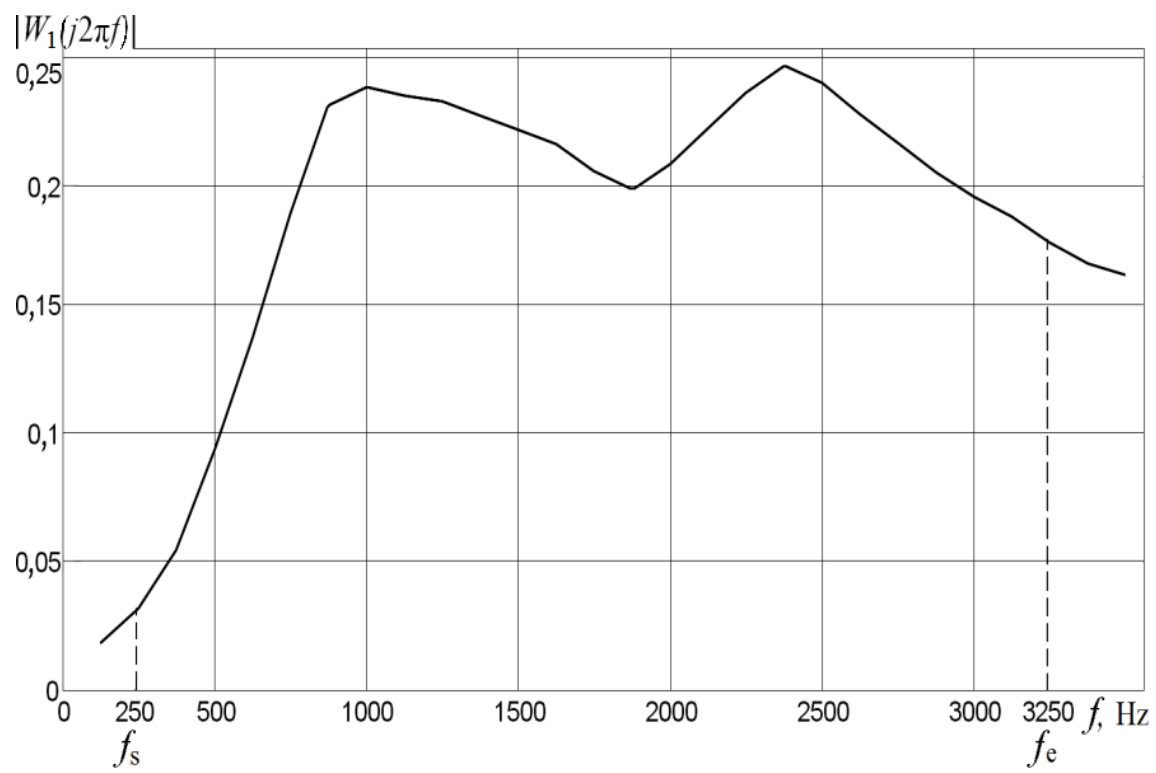

Figure 22. AFC of the first order after wavelet "Coiflet" $2^{\text {nd }}$ level noise-suppression

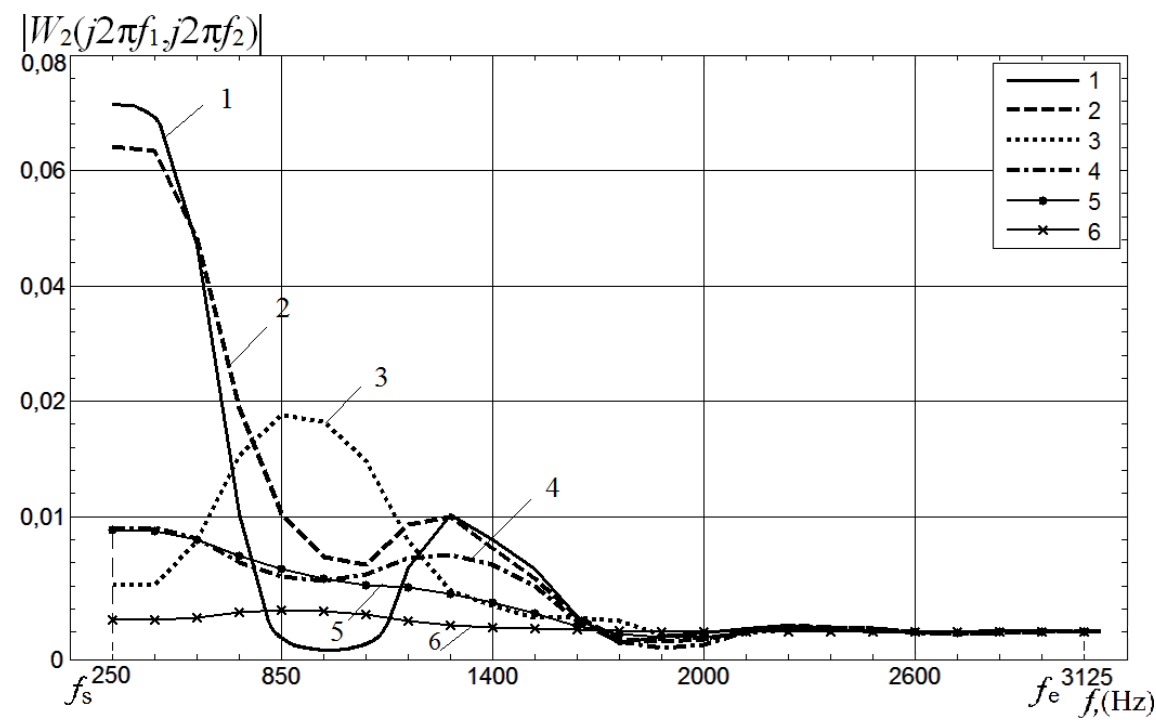

Figure 23. Subdiagonal sections of AFCs of the second order after wavelet "Coiflet" $2^{\text {nd }}$ level noise-suppression at different frequencies $F_{1}: 201$ (1), 401 (2), 601 (3), 801 (4), 1001 (5), 1401 (6) Hz 


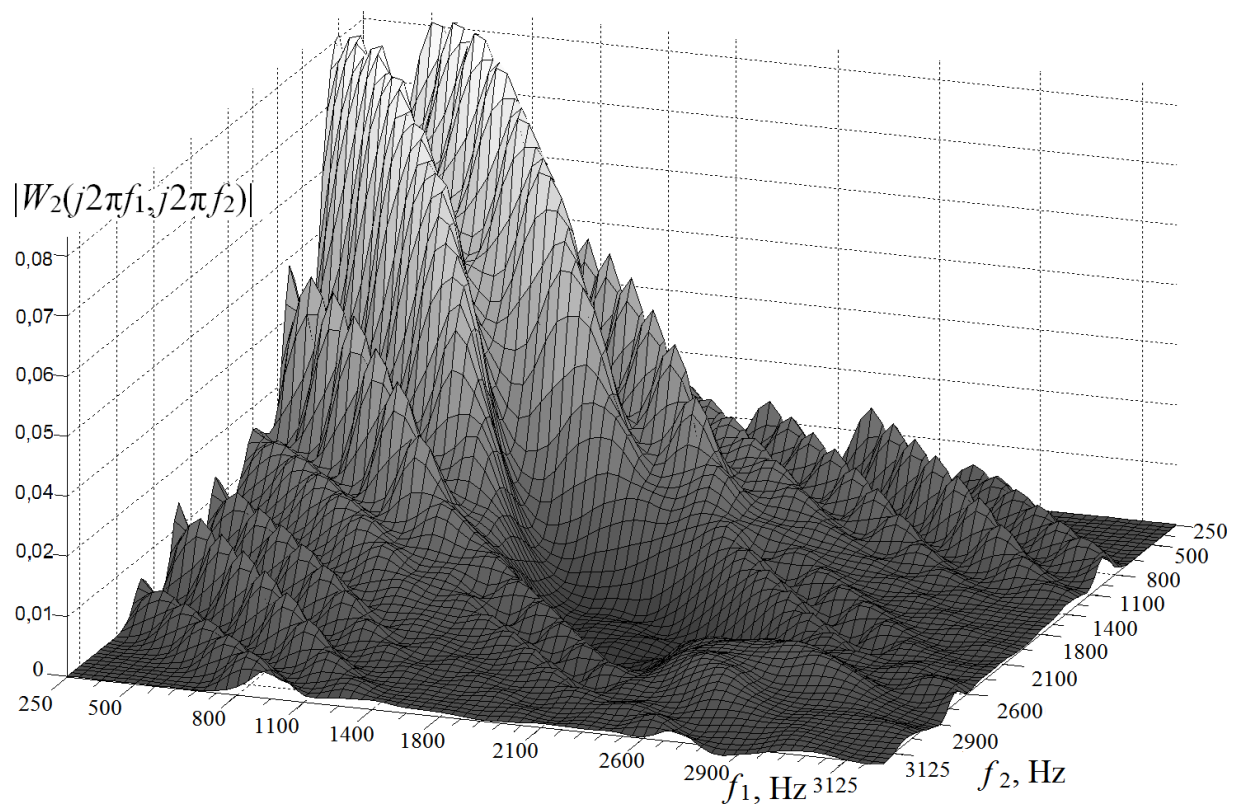

Figure 24. Surface built of AFCs of the second order after wavelet "Coiflet" $3^{\text {rd }}$ level noise-suppression

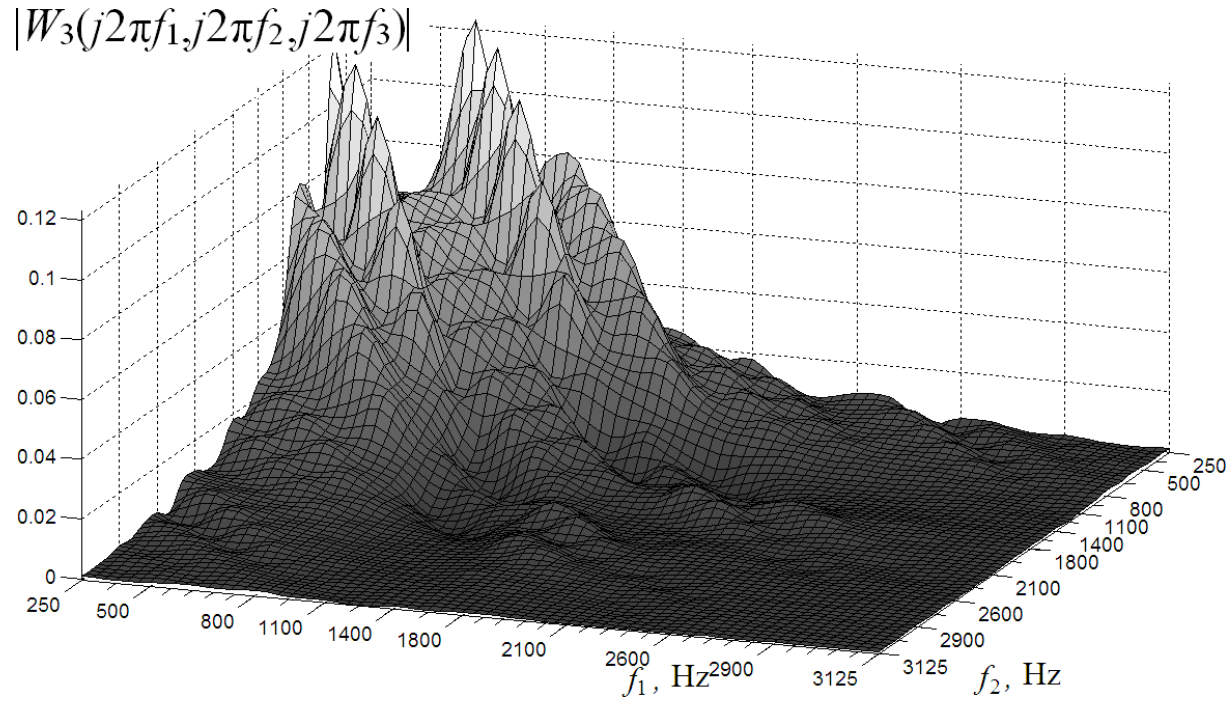

Figure 25. Surface built of AFCs of the third order after wavelet "Coiflet" $3^{\text {rd }}$ level noise-suppression, where $f_{3}=127,5 \mathrm{~Hz}$ 


\section{Conclusion}

Communication channel as a media for remote sensing systems functioning is analyzed. Nonlinear effects of the environments have great impact on result data received in experiments. The method based on Volterra model using polyharmonic test signals for identification nonlinear dynamical systems is analyzed. To differentiate the responses of system for partial components we use the method based on linear combination of responses on test signals with different amplitudes.

New values of test signals amplitudes were defined and they are greatly raising the accuracy of identification compared to amplitudes and coefficients written in [1]. The accuracy of identification of nonlinear part of the test system growth 2 times and the standard deviation in this case is about $5 \%$.

The interpolation method of identification using the hardware methodology written in [5] is applied for construction of informational Volterra model as an APC of the first and second order for UHF band radio channel.

Received results reveal essential nonlinearity of the CC that leads to distortions of signals in radio broadcasting devices, reduces the important indicators of the TCS: accuracy of signals reproduction, throughput, noise immunity.

The noise immunity is very high for the linear model, high enough for the second order nonlinear model and has moderate noise immunity for the third order model. The wavelet denoising is very effective and gives the possibility to improve the quality of identification of the noisy measurements up to 1,54 and 4,07 times for the AFC and PFC respectively.

Final characteristics of the CC have to be used to maintain sensor systems to improve the adequateness of received data.

\section{Author details}

Vitaliy Pavlenko and Viktor Speranskyy

Institute of Computer Systems, Odessa National Polytechnic University, Odessa, Ukraine

\section{References}

[1] Danilov LV, Mathanov PN, Philipov ES. The theory of nonlinear electrical circuits. Published Energoatomizdat, Leningrad; 1990. 
[2] Donoho DL, Johnstone IM. Threshold selection for wavelet shrinkage of noisy data. Proc. 16th Annual Conf. of the IEEE Engineering in Medicine and Biology Society, 24a-25a, IEEE Press; 1994.

[3] Doyle FJ, Pearson RK, Ogunnaike BA. Identification and Control Using Volterra Models. Published Springer Technology \& Industrial Arts; 2001.

[4] Giannakis GB, Serpedin EA. bibliography on nonlinear system identification and its applications in signal processing, communications and biomedical engineering. Signal Processing, EURASIP, Elsevier Science B.V. 81(3); 2001. p533-580.

[5] Goswami JG, Chan AK. Fundamentals of Wavelets: Theory, Algorithms, and Applications. Publishing John Wiley\&Sons Inc; 1999.

[6] Liew SC. Principles of remote sensing. Space View of Asia, 2nd Edition. CRISP; 2001. http://www.crisp.nus.edu.sg/ research/tutorial/rsmain.htm (accessed 1 November 2013).

[7] Misiti M, Misiti Y, Oppenheim G, Poggi J-M. Wavelets Toolbox Users Guide. The MathWorks. Wavelet Toolbox, for use with MATLAB; 2000.

[8] Marghany M. Volterra-Lax-wendroff algorithm for modelling sea surface flow pattern from Jason-1 satellite altimeter data. Lecture Notes in Computer Science (including subseries Lecture Notes in Artificial Intelligence and Lecture Notes in Bioinformatics) Volume 5730 LNCS; 2009; p1-18.

[9] Marghany M, Mazlan H, Cracknell AP. 3-D visualizations of coastal bathymetry by utilization of airborne TOPSAR polarized data. International Journal of Digital Earth; 3(2); p187-206.

[10] Marghany M. Three-Dimensional Coastal Front Visualization from RADARSAT-1 SAR Satellite Data. In Murgante B. et al. (eds.): Lecture Notes in Computer Science (ICCSA 2012); Part III, LNCS 7335; p447-456.

[11] Pavlenko V, Speranskyy V, Ilyin V, Lomovoy V. Modified Approximation Method for Identification of Nonlinear Systems Using Volterra Models in Frequency Domain. Applied Mathematics in Electrical and Computer Engineering. Proceedings of the AMERICAN-MATH'12 \& CSST'12 \& CEA'12, Harvard, Cambridge, USA, January 25-27, 2012. Published by WSEAS Press; 2012. p423-428.

[12] Pavlenko VD, Pavlenko SV, Speranskyy VO. Interpolation Method of Nonlinear Dynamical Systems Identification Based on Volterra Model in Frequency Domain. Proceedings of the 7th IEEE International Conference on Intelligent Data Acquisition and Advanced Computing Systems: Technology and Applications (IDAACS'2013), 15-17 September 2013, Berlin, Germany; 2013. p173-178.

[13] Pavlenko VD, Speranskyy VA. Analysis of identification accuracy of nonlinear system based on Volterra model in frequency domain. American Journal of Modeling and Optimization; Vol.1, No.2; 2013. p11-18. DOI: 10.12691/ajmo-1-2-2. 
[14] Pavlenko VD, Speranskyy VO. Communication Channel Identification in Frequency Domain Based on the Volterra Model. Recent Advances in Computers, Communications, Applied Social Science and Mathematics. Proceedings of the International Conference on Computers, Digital Communications and Computing (ICDCC'11), Barcelona, Spain, September 15-17, 2011. Published by WSEAS Press; 2011. p218-222.

[15] Pavlenko VD, Speranskyy VO. Interpolation method modification for nonlinear objects identification using Volterra model in frequency domain. 23rd International Crimean Conference "Microwave \& Telecommunication Technology" (CriMiCo'2013), Sevastopol, Ukraine; 2013. p257-260.

[16] Pavlenko VD, Speranskyy VO, Lomovoy VI. Modelling of Radio-Frequency Communication Channels Using Volterra Model. Proc. of the 6th IEEE International Conference on Intelligent Data Acquisition and Advanced Computing Systems: Technology and Applications (IDAACS'2011), 15-17 September 2011, Prague, Czech Republic; 2011. p574-579.

[17] Pavlenko VD, Speranskyy VO, Lomovoy VI. The Test Method for Identification of Radiofrequency Wireless Communication Channels Using Volterra Model. Proc. of the 9th IEEE East-West Design \& Test Symposium (EWDTS'2011), Sevastopol, Ukraine, September 9-12, 2011; Kharkov: KNURE; 2011. p331-334.

[18] Pavlenko V, Lomovoy V, Speranskyy V, Ilyin V. Radio frequency test method for wireless communications using Volterra model. Proc. of the 11th conference on dynamical systems theory and applications (DSTA'2011), December 5-8, 2011, Łódź, Poland; 2011. p446-452.

[19] Pavlenko VD, Speranskyy VO. Analysis of nonlinear system identification accuracy based on Volterra model in frequency domain. Electrotechnic and Computer Systems. Kiev: «Technica»; 08(84); 2012. p66-71.

[20] Pavlenko VD, Speranskyy VO. Identification of nonlinear dynamical systems using Volterra model with interpolation method in frequency domain. Electrotechnic and Computer Systems. Kiev: «Technica»; 2012. 05(81). p229-234.

[21] Pavlenko VD, Speranskyy VO. Simulation of Telecommunication Channel Using Volterra Model in Frequency Domain. 10th IEEE East-West Design \& Test Symposium (EWDTS'2012), Kharkov, Ukraine, September 14-17, 2012; 2012. p401-404.

[22] Schetzen M. The Volterra and Wiener Theories of Nonlinear Systems. Wiley\&Sons, New York; 1980.

[23] Westwick DT. Methods for the Identification of Multiple-Input Nonlinear Systems. Departments of Electrical Engineering and Biomedical Engineering, McGill University, Montreal, Quebec, Canada; 1995. 
